CERN-TH/98-194, CTP TAMU-27/98, Imperial/TP/97-98/57

LPTENS-98/19, SISSARef. 75/98/EP

hep-th/9807051

July 1998

\title{
Instanton Cosmology and Domain Walls from M-theory and String Theory
}

\author{
M.S. Bremer ${ }^{\star} 1$, M.J. Duff ${ }^{\dagger 2}$, H. Lü ${ }^{\ddagger}$, C.N. Pope ${ }^{\dagger \S} 3$ and K.S. Stelle ${ }^{\star \sharp}$ \\ * The Blackett Laboratory, Imperial College 4 \\ Prince Consort Road, London SW' 2BZ, UK \\ \# TH Division, CERN, CH-1211 Geneva 23, Switzerland \\ ${ }^{\dagger}$ Center for Theoretical Physics, Texas AEM University, College Station, Texas 77843 \\ ${ }^{\ddagger}$ Laboratoire de Physique Théorique de l'École Normale Supérieure ${ }^{4,5}$ \\ 24 Rue Lhomond - 75231 Paris CEDEX 05 \\ ${ }^{\S}$ SISSA, Via Beirut No. 2-4, 34013 Trieste, Italy 4
}

\begin{abstract}
$\underline{\text { ABSTRACT }}$
The recent proposal by Hawking and Turok for obtaining an open inflationary universe from singular instantons makes use of low-energy effective Lagrangians describing gravity coupled to scalars and non-propagating antisymmetric tensors. In this paper we derive some exact results for Lagrangians of this type, obtained from spherical compactifications of M-theory and string theory. In the case of the $S^{7}$ compactification of M-theory, we give a detailed discussion of the cosmological solutions. We also show that the lower-dimensional Lagrangians admit domain-wall solutions, which preserve one half of the supersymmetry, and which approach AdS spacetimes near their horizons.
\end{abstract}

\footnotetext{
${ }^{1}$ Research supported in part by the EC under TMR contract ERBFMBI-CT97-2344.

${ }^{2}$ Research supported in part by NSF Grant PHY-9722090.

${ }^{3}$ Research supported in part by DOE grant DE-FG03-95ER40917.

${ }^{4}$ Research supported in part by the EC under TMR contract ERBFMRX-CT96-0045.

${ }^{5}$ Unité Propre du Centre National de la Recherche Scientifique, associée à l'École Normale Supérieure et à l'Université de Paris-Sud.
} 


\section{Contents}

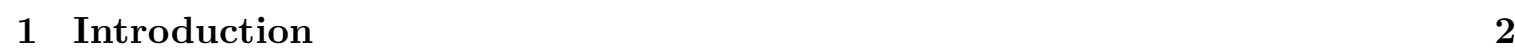

2 Sphere reductions of supergravities 4

$2.1 \quad$ Reduction of $D=11$ supergravity on $S \ldots \ldots \ldots \ldots$. . . . . . . 4

2.2 Inclusion of a squashing mode in the $S^{7}$ reduction . . . . . . . . . . 6

$2.3 \quad S^{4}$ reduction of $D=11 \ldots \ldots \ldots \ldots \ldots$

$2.4 \quad S^{5}$ reduction of type IIB supergravity $\ldots \ldots \ldots \ldots \ldots$

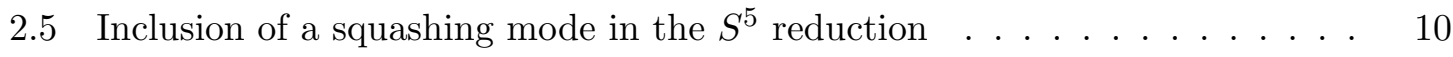

2.6 Further examples . . . . . . . . . . . . . . . . . . . . . 12

\begin{tabular}{|lll}
3 & Supersymmetric domain walls & 14
\end{tabular}

3.1 Domain wall in $D=4 \ldots \ldots \ldots \ldots$. . . . . . . . . . . . 14

3.2 Oxidation of domain walls . . . . . . . . . . . . . . . . . . . . 19

3.3 Further examples . . . . . . . . . . . . . . . . . . . . . . . 20

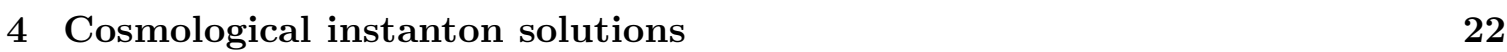

4.1 The Wick-rotated Euclidean-signature theory . . . . . . . . . . . . . . . . . 24

4.2 Patching cosmological solutions with domain walls . . . . . . . . . . . 30

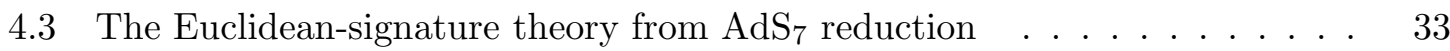

$\begin{array}{lll}5 & \text { Conclusions } & 36\end{array}$

\begin{tabular}{lr}
\hline Appendices & 39
\end{tabular}

\begin{tabular}{|ll}
\hline A General sphere and AdS reductions & 39
\end{tabular}

\begin{tabular}{|l|l|}
\hline B A general class of domain walls & 41
\end{tabular}

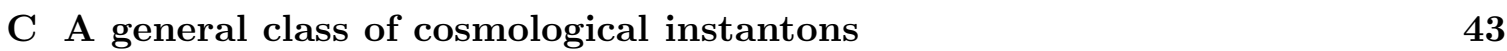

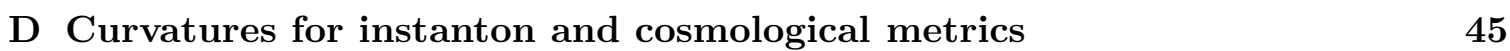

\begin{tabular}{|lll}
\hline E & Effective cosmological constants in sphere reductions & 46
\end{tabular} 


\section{Introduction}

It has recently been proposed that a Euclidean-signature instanton solution can describe the creation of an open inflationary universe [1]. The proposal has sparked a lively debate, in which various aspects of the assumptions and the conclusions have been discussed [2, 3, 4, 5, 6, 17, 8, 9, 10, 11, 12].

One of the hopes of string theory or M-theory is that it will be able to supply definitive answers to questions that until now have been matters for conjecture or choice. Indeed in [1] the form of the potential governing the evolution of an inflationary instanton solution was drawn from M-theory, or, more specifically, from the eleven-dimensional supergravity that is presumed to describe its low-energy limit, by compactifying the theory on a 7sphere. This makes use of the observation that a 4-form field strength can give rise to a cosmological constant [13, 14, 15, 16, 17, 18, 19]. Our purpose here is not to step out into the fray in the ongoing cosmological debate, but rather to study in more detail the lessons that can be learned by taking M-theory or string theory as a starting-point for the discussion. We shall consider various examples in which higher-dimensional supergravities are dimensionally reduced on spheres, leading to theories in lower dimensions that include scalar fields with the kind of potentials that are commonly encountered in the inflationary models. Of course, the complete analysis of the dimensionally-reduced theories is extremely complicated, even at the linearised level where one is simply concerned with extracting the mass spectrum. In fact for the purposes of studying cosmological solutions it is not sufficient to know just the linearised results, since the scalar fields are liable to become large at some stage in the evolution of the system. Thus it is of more interest to know the exact form of the lower-dimensional Lagrangian for some subset of the fields that includes the ones participating in the cosmological solution. In particular, if one wishes to retain comparability between the different dimensional theories, it is important that the truncation to the subset of fields in the lower-dimensional theory be a consistent one, in the sense that solutions of the resulting lower-dimensional equations of motion should also give solutions of the original higher-dimensional ones.

The easiest way of achieving a consistent truncation in any system of equations is to retain the totality of fields that are singlets under some symmetry group, while setting to zero all fields that are non-singlets [20]. By this means the danger inherent in any "generic" truncation, namely that non-linear terms can imply that the retained fields act as sources for the fields that were set to zero, is avoided.] We shall consider a number

\footnotetext{
${ }^{1}$ In the context of a supergravity theory, one also encounters many fields transforming nontrivially under
} 
of examples, based on sphere compactifications of higher-dimensional supergravities. The simplest cases are where the theory is compactified on a "round" n-sphere with $S O(n+1)$ isometry group, and the dimensionally-reduced theory is truncated to the $S O(n+1)$ singlets. In particular, the Einstein-Hilbert sector of the higher-dimensional theory will give rise just to the metric, and a "breathing-mode" scalar in the lower dimension. Slightly more complicated examples can be found in cases where the dimension $n$ of the sphere $S^{n}$ is odd, $n=2 m+1$. In such cases, we may truncate to the larger subsector of all singlets under the $S U(m+1) \times U(1)$ subgroup of $S O(2 m+2)$. This is the isometry group of the one-parameter family of homogeneous metrics on the "squashed" $(2 m+1)$-sphere, which can be described as a $U(1)$ bundle over $C P^{m}$. (The squashing parameter corresponds to the freedom to scale the length of the $U(1)$ fibres, without affecting the isometry group. For one particular value of this parameter, corresponding to the "round" sphere, the isometry group undergoes an enlargement from $S U(m+1) \times U(1)$ to $S O(2 m+2)$.) Thus we may construct consistently-truncated lower-dimensional theories that include the homogeneous squashing mode as well as the breathing mode of the sphere.

In this paper, we shall construct consistently-truncated theories that result from spherical reductions in a number of cases, including the round and squashed $S^{7}$ reductions of $D=11$ supergravity, the $S^{4}$ reduction of $D=11$ supergravity, and the round and squashed $S^{5}$ reductions of type IIB supergravity. Owing to the presence of cosmological potentials in these dimensionally reduced theories, there is a salient class of supersymmetric solutions containing $(D-2)$ branes, i.e. domain walls. Unlike the domain walls occurring in massive supergravities [33, 34], these domain walls have regular dilatons on their horizons. Thus, they fit into the class of solitons interpolating between different vacua of the dimensionally reduced theory, specifically, between anti-de Sitter space and flat space. Accordingly, they also display supersymmetry enhancement at their horizons. We shall show that these domain-wall solutions can be interpreted via dimensional oxidation on spheres, in terms of the familiar fundamental brane solutions of $D=11$ and $D=10$ type IIB supergravities. Next, we proceed to consider instanton solutions obtained in two variants of Euclidean supergravities: those obtained by Wick rotation and those obtained by dimensional reduction on anti-de Sitter spaces, a Minkowski-signature variant of the spherical reductions considered earlier. In the Wick-rotated $D=4$ theory obtained via an $S^{7}$ reduction, we obtain internal symmetry groups. Although such fields will not play a rôle in the specific solutions that will occupy us in the present paper, it is appropriate to note that truncation to the full supergravity multiplet is also consistent [21,22]. 
an instanton that shares some features with the Hawking-Turok instanton [1], but now obtained as a specific solution of Euclideanised $D=11$ supergravity. Moreover, we shall see that one can also follow the spirit of Ref. 41] and combine domain walls and instantons together in a nonsingular solution with both Euclidean and Minkowskian regions. Although we have not carried out a full analysis of the cosmological implications of such solutions, we note that the occurrence of domain walls and instantons within these dimensionally reduced supergravities appears to provide some of the elements postulated in recent cosmological discussions, but now within the definite context of supergravity theory.

\section{Sphere reductions of supergravities}

In this section, we consider the consistent reductions of various supergravity theories on spheres, using the general results obtained in appendix A. Specifically, we shall consider the cases of $D=11$ supergravity compactified on $S^{7}$ and on $S^{4}$, type IIB supergravity compactified on $S^{5}, D=6$ supergravity on $S^{3}$, and $D=5$ supergravity on $S^{2}$. In all the cases where the internal space is a sphere of odd dimension, one can also retain a further singlet mode in a consistent truncation, namely the "squashing" mode parameterising the length of the $U(1)$ fibres in the description of $S^{2 m+1}$ as a $U(1)$ bundle over $C P^{m}$. In this case, the modes that are retained will be singlets under the $S U(m+1) \times U(1)$ subgroup of $S O(2 m+2)$ that is the isometry group of the generic squashed sphere. As discussed in appendix $\mathrm{A}$, the results are also easily generalised to the case of timelike reductions on AdS spaces instead of spheres. In these cases the breathing-mode potential in the lowerdimensional Lagrangian is reversed in sign. Note also that all the results for sphere and AdS reductions apply equally well to any other internal Einstein spaces, with positive, negative or zero Ricci scalar.

\subsection{Reduction of $D=11$ supergravity on $S^{7}$}

Following the general discussion in appendix A, we reduce the eleven-dimensional fields $\hat{g}_{M N}$ and $\hat{F}_{4}$ on $S^{7}$ :

$$
\begin{aligned}
& d \hat{s}^{2}=e^{2 \alpha \varphi} d s_{4}^{2}+e^{2 \beta \varphi} d s_{7}^{2}, \\
& \hat{F}_{(4)}=F_{(4)} .
\end{aligned}
$$

Here, the metric $d s_{4}^{2}$ and the fields $F_{(4)}$ and $\varphi$ depend only on the four coordinates of the four-dimensional space, whereas the hatted fields depend, a priori, on all eleven coordinates. 
The metric $d s_{7}^{2}$ is a fixed metric on the round 7 -sphere, with a fixed standard radius. The constants $\alpha$ and $\beta$ are given by

$$
\alpha=\frac{\sqrt{7}}{6}, \quad \beta=-\frac{2}{7} \alpha
$$

We then obtain the $D=4$ Lagrangian

$$
e^{-1} \mathcal{L}=R-\frac{1}{2}(\partial \varphi)^{2}+e^{\frac{18 \alpha}{7} \varphi} R_{7}-\frac{1}{48} e^{-6 \alpha \varphi} F_{(4)}^{2}
$$

Here, $R_{7}$ is the Ricci scalar of the 7 -sphere; it is just a fixed constant.

The four-dimensional field equations following from this Lagrangian are

$$
\begin{aligned}
& R_{\mu \nu}=\frac{1}{2} \partial_{\mu} \varphi \partial_{\nu} \varphi+\frac{1}{12} e^{-6 \alpha \varphi}\left(F_{(4) \mu \nu}^{2}-\frac{3}{8} F_{(4)}^{2} g_{\mu \nu}\right)-\frac{1}{2} e^{\frac{18 \alpha}{7} \varphi} R_{7} g_{\mu \nu} \\
& \square \varphi=-\frac{18 \alpha}{7} e^{\frac{18 \alpha}{7} \varphi} R_{7}-\frac{\alpha}{8} e^{-6 \alpha \varphi} F_{(4)}^{2} \\
& \nabla^{\mu}\left(e^{-6 \alpha \varphi} F_{\mu \nu \rho \sigma}\right)=0 .
\end{aligned}
$$

The last equation implies that $F_{4}$ can be solved by writing

$$
F_{\mu \nu \rho \sigma}=c e^{6 \alpha \varphi} \epsilon_{\mu \nu \rho \sigma}
$$

where $c$ is a constant. Either by substituting this back into the field equations, or by following the standard procedure for dualising a field, we can obtain the Lagrangian describing the dualised system

$$
e^{-1} \mathcal{L}=R-\frac{1}{2}(\partial \varphi)^{2}-V(\varphi)
$$

where the potential $V(\varphi)$ is given by

$$
V(\varphi)=\frac{1}{2} c^{2} e^{6 \alpha \varphi}-e^{\frac{18 \alpha}{7} \varphi} R_{7} .
$$

The Lagrangian (2.6) is a special case of (A.13) given in appendix A.

It can be seen that this $D=4$ theory admits a solution where $\varphi=\varphi_{\text {AdS }}$ is a constant. The Ricci scalar $R_{7}$ of the 7 -sphere is then given in terms of $c$ and $\varphi_{\text {AdS }}$. This includes the $\mathrm{AdS}_{4} \times S^{7}$ solution. Specifically, we will have:

$$
\begin{aligned}
R_{\mu \nu} & =-\frac{1}{3} c^{2} e^{6 \alpha \varphi_{\mathrm{AdS}}} g_{\mu \nu} \\
e^{\frac{24 \alpha}{7} \varphi_{\mathrm{AdS}}} & =\frac{6 R_{7}}{7 c^{2}}
\end{aligned}
$$

Later in the paper, we shall obtain a domain-wall solution of this theory that interpolates between this "vacuum" solution and flat space. We shall also show that this four-dimensional theory admits cosmological instanton solutions, similar to those described in [1]. 


\subsection{Inclusion of a squashing mode in the $S^{7}$ reduction}

Another consistent truncation of the $S^{7}$ reduction from $D=11$ can be obtained by retaining all the singlets under the $S U(4) \times U(1)$ subgroup of $S O(8)$, rather than just the $\mathrm{SO}(8)$ singlets. Since $S U(4) \times U(1)$ is the isometry group of the one-parameter family of 7 -spheres constructed as $U(1)$ bundles over $C P^{3}$, for generic values of the "squashing parameter" along the $U(1)$ fibres, it follows that the $D=4$ scalar field corresponding to this squashing mode is a singlet under $S U(4) \times U(1)$. In fact, we can treat this problem by viewing the reduction on $S^{7}$ as a reduction first to $D=10$ type IIA, followed by a reduction on $C P^{3}$, in which we keep just the singlets under the $S U(4)$ isometry group of $C P^{3}$. Thus the full set of fields in $D=4$ that are singlets under $S U(4) \times U(1)$ will be the direct reduction of the fields already present in $D=10$ type IIA, plus the further scalar field corresponding to the "breathing mode" of $C P^{3} \cdot[$

To establish notation, let us write the ansatz for the $D=11$ metric reduced to $D=10$ as

$$
d s_{11}^{2}=e^{-\frac{1}{6} \phi} d s_{10}^{2}+e^{\frac{4}{3} \phi}(d z+\mathcal{A})^{2} .
$$

Note that $\phi$ is nothing but the dilaton of the type IIA theory. In this convention, the ten-dimensional string coupling constant is given by $\lambda_{10}=e^{\phi}$. Then the reduction on $C P^{3}$ from $D=10$ to $D=4$ will be performed as follows:

$$
d s_{10}^{2}=e^{2 \alpha \varphi} d s_{4}^{2}+e^{2 \beta \varphi} d s^{2}\left(C P^{3}\right) .
$$

This part of the reduction proceeds identically to the sphere reductions discussed in the previous section. (No special features of the sphere metric were needed in that discussion; we can equally well apply the previous results to reductions on any Einstein metric.) Thus we will have

$$
\alpha=\frac{\sqrt{3}}{4}, \quad \beta=-\frac{1}{3} \alpha
$$

in this case. The volume of the $C P^{3}$ measured in the ten-dimensional string frame is given by

$$
V_{C P^{3}}=e^{\frac{3}{2} \phi+6 \beta \varphi}
$$

Thus the four-dimensional string coupling constant is given by

$$
\lambda_{4}^{2}=\frac{\lambda_{10}^{2}}{V_{C P^{3}}}=e^{\frac{1}{2} \phi+\frac{\sqrt{3}}{2} \varphi} .
$$

\footnotetext{
${ }^{2}$ A similar kind of analysis for the squashing of $S^{7}$ described as an $S U(2)$ bundle over $S^{4}$, which arises in the $N=1$ supersymmetric $\mathrm{AdS}_{4} \times S^{7}$ "squashed seven-sphere" compactification of $D=11$ supergravity 23. can be found in 24.
} 
The various fields in $D=10$ will be reduced as follows:

$$
\begin{aligned}
& \hat{F}_{(4)}=F_{(4)}, \quad \hat{F}_{(3)}=F_{(3)}, \\
& \hat{\mathcal{F}}_{(2)}=\mathcal{F}_{(2)}+2 m J,
\end{aligned}
$$

where $J$ is the Kähler form on $C P^{3}$. In other words, $\hat{F}_{(4)}$ and $\hat{F}_{(3)}$ are just taken to be independent of the $C P^{3}$ coordinates, while $\hat{\mathcal{F}}_{(2)}$ is taken to have its background value as in the $\mathrm{AdS}_{4} \times S^{7}$ solution (see [25]), plus a fluctuation. The final result for the reduced Lagrangian in $D=4$ will therefore be

$$
\begin{aligned}
e^{-1} \mathcal{L}= & R-\frac{1}{2}(\partial \phi)^{2}-\frac{1}{2}(\partial \varphi)^{2}+e^{\frac{8}{3} \alpha \varphi} R_{6}-6 m^{2} e^{\frac{3}{2} \phi+\frac{10 \alpha}{3} \varphi} \\
& -\frac{1}{48} e^{\frac{1}{2} \phi-6 \alpha \varphi} F_{(4)}^{2}-\frac{1}{12} e^{-\phi-4 \alpha \varphi} F_{(3)}^{2}-\frac{1}{4} e^{\frac{3}{2} \phi-2 \alpha \varphi} \mathcal{F}_{(2)}^{2},
\end{aligned}
$$

where $R_{6}$ is the Ricci scalar of the compactifying $C P^{3}$ space.

A simple check is to verify that we can recover the usual $\mathrm{AdS}_{4} \times S^{7}$ solution of $D=11$ supergravity. Now, from (2.15), we have that the equations of motion for the two dilatons and the metric are

$$
\begin{aligned}
\square \phi= & 9 m^{2} e^{\frac{3}{2} \phi+\frac{10 \alpha}{3} \varphi}+\frac{1}{96} e^{\frac{1}{2} \phi-6 \alpha \varphi} F_{(4)}^{2}-\frac{1}{12} e^{-\phi-4 \alpha \varphi} F_{(3)}^{2}+\frac{3}{8} e^{\frac{3}{2} \phi-2 \alpha \varphi} \mathcal{F}_{(2)}^{2}, \\
\square \varphi= & -\frac{8}{3} \alpha R_{6} e^{\frac{8 \alpha}{3} \varphi}+20 \alpha m^{2} e^{\frac{3}{2} \phi+\frac{10 \alpha}{3} \varphi}-\frac{\alpha}{8} e^{\frac{1}{2} \phi-6 \alpha \varphi} F_{(4)}^{2} \\
& -\frac{\alpha}{3} e^{-\phi-4 \alpha \varphi} F_{(3)}^{2}-\frac{\alpha}{2} e^{\frac{3}{2} \phi-2 \alpha \varphi} \mathcal{F}_{(2)}^{2}, \\
R_{\mu \nu}= & \frac{1}{2} \partial_{\mu} \phi \partial_{\nu} \phi+\frac{1}{2} \partial_{\mu} \varphi \partial_{\nu} \varphi-\frac{1}{2} R_{6} e^{\frac{8 \alpha}{3} \varphi} g_{\mu \nu}+3 m^{2} e^{\frac{3}{2} \phi+\frac{10 \alpha}{3} \varphi} g_{\mu \nu} \\
& +\frac{1}{12} e^{\frac{1}{2} \phi-6 \alpha \varphi}\left(F_{(4) \mu \nu}^{2}-\frac{3}{8} F_{(4)}^{2} g_{\mu \nu}\right)+\frac{1}{4} e^{-\phi-4 \alpha \varphi}\left(F_{(3) \mu \nu}^{2}-\frac{1}{3} F_{(3)}^{2} g_{\mu \nu}\right) \\
& +\frac{1}{2} e^{\frac{3}{2} \phi-2 \alpha \varphi}\left(\mathcal{F}_{(2) \mu \nu}^{2}-\frac{1}{4} \mathcal{F}_{(2)}^{2} g_{\mu \nu}\right) .
\end{aligned}
$$

We see that there is indeed a solution where $F_{(4)}=c e^{-\frac{1}{2} \phi+6 \alpha \varphi} \epsilon_{(4)}$, and with $\phi=\phi_{0}$ and $\varphi=\varphi_{0}$ constants given by

$$
e^{2 \phi_{0}}=\sqrt{\frac{c}{6 m}} \frac{R_{6}}{48 m^{2}}, \quad e^{\frac{16 \alpha}{3} \varphi_{0}}=\frac{3 R_{6}^{2}}{32 m c^{2}} .
$$

The Ricci tensor satisfies

$$
R_{\mu \nu}=-\frac{1}{4} R_{6} e^{\frac{8 \alpha}{3} \varphi_{0}} g_{\mu \nu},
$$

which admits, in particular, the standard $\mathrm{AdS}_{4}$ solution.

Another check is to see that we can obtain the $S O(8)$-invariant truncation of section 2.1 in a consistent way. To do this, we can perform a rotation of the dilatons in the case above, and define a pair $(\tilde{\phi}, \tilde{\varphi})$ as follows:

$$
\begin{aligned}
\tilde{\phi} & =\frac{3 \sqrt{3}}{2 \sqrt{7}} \phi+\frac{1}{2 \sqrt{7}} \varphi \\
\tilde{\varphi} & =-\frac{1}{2 \sqrt{7}} \phi+\frac{3 \sqrt{3}}{2 \sqrt{7}} \varphi .
\end{aligned}
$$


Substituting into (2.15), and dropping the fields $F_{(3)}$ and $\mathcal{F}_{(2)}$, we obtain

$$
\begin{aligned}
e^{-1} \mathcal{L}= & R-\frac{1}{2}(\partial \tilde{\phi})^{2}-\frac{1}{2}(\partial \tilde{\varphi})^{2}+R_{6} e^{\frac{3}{\sqrt{7}} \tilde{\varphi}+\frac{1}{\sqrt{21}} \tilde{\phi}}-6 m^{2} e^{\frac{3}{\sqrt{7}} \tilde{\varphi}+\frac{8}{\sqrt{21}} \tilde{\phi}} \\
& -\frac{1}{48} e^{-\sqrt{7} \tilde{\varphi}} F_{(4)}^{2} .
\end{aligned}
$$

We see that the equation of motion for $\tilde{\phi}$ allows us to set $\tilde{\phi}=0$, provided that $R_{6}=48 \mathrm{~m}^{2}$, whereupon the Lagrangian (2.20) reduces to the Lagrangian (2.3) of section 2.1 (with $R_{7}=$ $42 \mathrm{~m}^{2}$ ), with $\varphi$ replaced by $\tilde{\varphi}$. Furthermore, if we write the ansatz $2.9,2.10$ ) for the elevendimensional metric in terms of the rotated tilde basis for the dilatons, we have

$$
d s_{11}^{2}=e^{\frac{\sqrt{7}}{3} \tilde{\varphi}} d s_{4}^{2}+e^{-\frac{2}{3 \sqrt{7}} \tilde{\varphi}}\left(e^{-\frac{1}{\sqrt{21}} \tilde{\phi}} d s^{2}\left(C P^{3}\right)+e^{\frac{6}{\sqrt{21}} \tilde{\phi}}\left(d z+\mathcal{A}_{(1)}\right)^{2}\right)
$$

Thus we see that indeed the $\tilde{\phi}$ scalar describes a volume-preserving $6+1$ squashing of the $S^{7}$ metric, while $\tilde{\varphi}$ is the same as the dilatonic scalar $\varphi$ of the $S O(8)$-invariant ansatz of section 2.1. Thus we see that the four-dimensional string coupling constant $\lambda_{4}$, given in (2.13) is not an $S O(8)$-invariant quantity. To get a four-dimensional theory with $\mathrm{AdS}_{4}$ background, we need to set $\tilde{\phi}=0$, implying that $\lambda_{4}=\lambda_{10}^{-2}$ [25].

\section{$2.3 S^{4}$ reduction of $D=11$}

In this case, the $S^{4}$ solution requires that the internal components of $F_{4}$ (in the 4 -sphere) should be non-zero, and so we must make the following ansatz:

$$
\begin{aligned}
d \hat{s}_{11}^{2} & =e^{2 \alpha \varphi} d s_{7}^{2}+e^{2 \beta \varphi} d s_{4}^{2} \\
\hat{F}_{(4)} & =F_{(4)}+6 m \epsilon_{(4)} .
\end{aligned}
$$

From appendix A, we see that the constants $\alpha$ and $\beta$ are given by $\alpha=\frac{2}{3 \sqrt{10}}$ and $\beta=-\frac{5}{4} \alpha$. Plugging into the Lagrangian, we get

$$
\mathcal{L}_{7}=e R-\frac{1}{2} e(\partial \varphi)^{2}+e e^{\frac{9 \alpha}{2} \varphi} R_{4}-18 e m^{2} e^{12 \alpha \varphi}-\frac{1}{48} e e^{-6 \alpha \varphi} F_{(4)}^{2}-3 m *\left(F_{(4)} \wedge A_{(3)}\right) .
$$

Note that we have a topological mass term for $A_{(3)}$ here, coming from the $F F A$ term in $D=11$.

The equations of motion following from this Lagrangian are

$$
\begin{aligned}
R_{\mu \nu}= & \frac{1}{2} \partial_{\mu} \varphi \partial_{\nu} \varphi-\frac{1}{5} e^{\frac{9 \alpha}{2} \varphi} R_{4} g_{\mu \nu}+\frac{18}{5} m^{2} e^{12 \alpha \varphi} g_{\mu \nu} \\
& +\frac{1}{12} e^{-6 \alpha \varphi}\left(F_{\mu \nu}^{2}-\frac{1}{10} F_{(4)}^{2} g_{\mu \nu}\right) \\
\square \varphi= & -\frac{9}{2} \alpha e^{\frac{9 \alpha}{2} \varphi} R_{4}+216 \alpha m^{2} e^{12 \alpha \varphi}-\frac{1}{8} \alpha e^{-6 \alpha \varphi} F_{(4)}^{2}, \\
d\left(e^{-6 \alpha \varphi} * F_{(4)}\right)= & -6 m F_{(4)} .
\end{aligned}
$$


These equations admit an $\mathrm{AdS}_{7}$ solution which corresponds to the $\operatorname{AdS}_{7} \times S^{4}$ "vacuum" solution of $D=11$ supergravity. We see from (2.24) that with $F_{(4)}=0$, we can take $\varphi=\varphi_{\text {AdS }}$ to be constant, given by

$$
e^{\frac{15 \alpha}{2} \varphi_{\mathrm{AdS}}}=\frac{R_{4}}{48 m^{2}}
$$

The Ricci tensor in spacetime is then given by

$$
R_{\mu \nu}=-6 m^{2} e^{12 \alpha \varphi_{\text {AdS }}} g_{\mu \nu}
$$

which allows an $\mathrm{AdS}_{7}$ solution.

\section{$2.4 S^{5}$ reduction of type IIB supergravity}

In the case of type IIB supergravity [26], we must work at the level of the $D=10$ equations of motion, since there there is no Lagrangian formulation of the theory. The type IIB bosonic equations of motion can be written as the following [27]

$$
\begin{aligned}
R_{\mu \nu}= & \frac{1}{2} \partial_{\mu} \phi \partial_{\nu} \phi+\frac{1}{2} e^{2 \phi} \partial_{\mu} \chi \partial_{\nu} \chi+\frac{1}{96}\left(H_{(5)}\right)_{\mu \nu}^{2} \\
& +\frac{1}{4} e^{\phi}\left(\left(F_{(3)}^{1}\right)_{\mu \nu}^{2}-\frac{1}{12}\left(F_{(3)}^{1}\right)^{2} g_{\mu \nu}\right)+\frac{1}{4} e^{-\phi}\left(\left(F_{(3)}^{2}\right)_{\mu \nu}^{2}-\frac{1}{12}\left(F_{(3)}^{2}\right)^{2} g_{\mu \nu}\right) \\
d\left(\mathcal{M} * H_{(3)}\right)= & H_{(5)} \wedge \Xi H_{(3)} \\
H_{(5)}= & * H_{(5)} \\
d\left(e^{2 \phi} * d \chi\right)= & -e^{\phi} F_{(3)}^{2} \wedge F_{(3)}^{1} \\
d * d \phi= & e^{2 \phi} d \chi \wedge * d \chi+\frac{1}{2} e^{\phi} F_{(3)}^{1} \wedge F_{(3)}^{1}-\frac{1}{2} e^{-\phi} F_{(3)}^{2} \wedge F_{(3)}^{2}
\end{aligned}
$$

where

$$
\mathcal{M}=\left(\begin{array}{cc}
e^{\phi} & \chi e^{\phi} \\
\chi e^{\phi} & e^{-\phi}+\chi^{2} e^{\phi}
\end{array}\right), \quad H_{(3)}=\left(\begin{array}{c}
d A_{(2)}^{1} \\
d A_{(2)}^{2}
\end{array}\right), \quad \Xi=\left(\begin{array}{cc}
0 & 1 \\
-1 & 0
\end{array}\right) .
$$

The self-dual 5-form $H_{(5)}$ satisfies the Bianchi identity $d H_{(5)}+\frac{1}{2} \epsilon_{i j} F_{(3)}^{i} \wedge F_{(3)}^{j}=0$. The R-R field strength $F_{(3)}^{1}$ is given by $F_{(3)}^{1}=d A_{(2)}^{1}-\chi d A_{(2)}^{1}$, and the NS-NS field strength is given by $F_{(3)}^{2}=d A_{(2)}^{2}$.

The Kaluza-Klein ansatz for the metric will be the usual one, invariant under the isometry group $S O(6)$ of the compactifying 5-sphere:

$$
d s_{10}^{2}=e^{2 \alpha \varphi} d s_{5}^{2}+e^{2 \beta \varphi} d s^{2}\left(S^{5}\right) .
$$

From the general results in appendix A, we have here that

$$
\alpha=\frac{1}{4} \sqrt{\frac{5}{3}} \quad \beta=-\frac{3}{5} \alpha .
$$


The ansatz for the self-dual 5-form, which is non-dynamical in the reduced $D=5$ theory, will be

$$
H_{(5)}=4 m e^{8 \alpha \varphi} \epsilon_{(5)}+4 m \epsilon_{(5)}\left(S^{5}\right)
$$

where the $\epsilon_{(5)}$ quantities are the volume forms on the five-dimensional spacetime and the $S^{5}$ metrics $d s_{5}^{2}$ and $d s_{5}^{2}\left(S^{5}\right)$ respectively. The choice of ansatz here is dictated by the requirements that the 5 -form be self-dual, and that it satisfy the necessary Bianchi identity $d H_{(5)}=0$. After some algebra, we find that substituting these ansätze into the tendimensional equations of motion leads consistently to the the 5 -dimensional equations

$$
\begin{aligned}
R_{\mu \nu}= & \frac{1}{2} \partial_{\mu} \phi \partial_{\nu} \phi+\frac{1}{2} e^{2 \phi} \partial_{\mu} \chi \partial_{\nu} \chi+\frac{8}{3} m^{2} e^{8 \alpha \varphi} g_{\mu \nu}-\frac{1}{3} e^{\frac{16 \alpha}{5} \varphi} R_{5} g_{\mu \nu} \\
& +\frac{1}{4} e^{\phi}\left(\left(F_{(3)}^{1}\right)_{\mu \nu}^{2}-\frac{2}{9}\left(F_{(3)}^{1}\right)^{2} g_{\mu \nu}\right)+\frac{1}{4} e^{-\phi}\left(\left(F_{(3)}^{2}\right)_{\mu \nu}^{2}-\frac{2}{9}\left(F_{(3)}^{2}\right)^{2} g_{\mu \nu}\right), \\
\square \varphi= & 64 \alpha m^{2} e^{8 \alpha \varphi}-\frac{16}{5} \alpha e^{\frac{16 \alpha}{5} \varphi} R_{5}-\frac{1}{3} \alpha e^{-\phi-4 \alpha \varphi}\left(F_{(3)}^{1}\right)^{2}-\frac{1}{3} \alpha e^{\phi-4 \alpha \varphi}\left(F_{(3)}^{2}\right)^{2} .
\end{aligned}
$$

(The other equations of motion do not immediately concern us here.) It is not hard to see that the full set of equations of motion can be derived from the 5-dimensional Lagrangian

$$
\begin{aligned}
\mathcal{L}_{5}= & e R-\frac{1}{2} e(\partial \phi)^{2}-\frac{1}{2} e(\partial \varphi)^{2}-\frac{1}{2}(\partial \chi)^{2} e^{2 \phi}-8 m^{2} e e^{8 \alpha \varphi}+e^{\frac{16 \alpha}{5} \varphi} R_{5} \\
& -\frac{1}{12} e e^{\phi-4 \alpha \varphi}\left(F_{(3)}^{1}\right)^{2}-\frac{1}{12} e e^{-\phi-4 \alpha \varphi}\left(F_{(3)}^{2}\right)^{2}-2 m *\left(\epsilon_{i j} A_{(2)}^{i} \wedge d A_{(2)}^{j}\right) .
\end{aligned}
$$

Note that this reduction allows the expected $\mathrm{AdS}_{5}$ solution, with $\varphi=\varphi_{\text {AdS }}$ being constant and given by

$$
e^{\frac{24 \alpha}{5} \varphi_{\text {AdS }}}=\frac{R_{5}}{20 m^{2}}
$$

The Ricci tensor for $\mathrm{AdS}_{5}$ is then given by

$$
R_{\mu \nu}=-4 m^{2} e^{8 \alpha \varphi_{\text {AdS }}} g_{\mu \nu}
$$

\subsection{Inclusion of a squashing mode in the $S^{5}$ reduction}

In the same manner as we did previously for $S^{7}$, we can include a squashing mode in the reduction of Type IIB supergravity on $S^{5}$, in which we view $S^{5}$ as a $U(1)$ bundle over $C P^{2}$, and thus we now truncate so as to keep all the $S U(3) \times U(1)$ singlets in the decomposition of the $S O(6)$ isometry group of the round $S^{5}$ under the $S U(3) \times U(1)$ subgroup that is the symmetry group of the squashed family of $S^{5}$ metrics. We may use a similar trick as in section 2.2, and carry out the process by first considering the $S^{1}$-reduced $D=9$ metric, and then reducing this on $C P^{2}$, while giving the appropriate background value to the KaluzaKlein 2-form field strength in $D=9$. We shall express the fields of the $D=9$ theory in the 
type IIB language, since our aim is to describe the squashed- $S^{5}$ reduction of the Type IIB theory.

The $D=9$ Lagrangian, obtained from the type IIB theory by reducing the metric according to the usual ansatz

$$
d s_{10}^{2}=e^{-\frac{1}{2 \sqrt{7}} \varphi} d s_{9}^{2}+e^{\frac{\sqrt{7}}{2} \varphi}\left(d z+\mathcal{A}_{(1)}\right)^{2}
$$

is

$$
\begin{aligned}
e^{-1} \mathcal{L}_{9}= & R-\frac{1}{2}(\partial \phi)^{2}-\frac{1}{2}(\partial \varphi)^{2}-\frac{1}{2} e^{2 \phi}(\partial \chi)^{2} \\
& -\frac{1}{48} e^{-\frac{2}{\sqrt{7}} \varphi} F_{(4)}^{2}-\frac{1}{12} e^{-\phi+\frac{1}{\sqrt{7}} \varphi}\left(F_{(3)}^{(\mathrm{NS})}\right)^{2}-\frac{1}{2} e^{\phi+\frac{1}{\sqrt{7}} \varphi}\left(F_{(3)}^{(\mathrm{R})}\right)^{2} \\
& -\frac{1}{4} e^{\frac{4}{\sqrt{7}} \varphi}\left(\mathcal{F}_{(2)}\right)^{2}-\frac{1}{4} e^{\phi-\frac{3}{\sqrt{7}} \varphi}\left(F_{(2)}^{(\mathrm{R})}\right)^{2}-\frac{1}{4} e^{-\phi-\frac{3}{\sqrt{7}} \varphi}\left(F_{(2)}^{(\mathrm{NS})}\right)^{2} \\
& -\frac{1}{2 e} d A_{(3)} \wedge d A_{(3)} \wedge \mathcal{A}_{(1)}+\frac{1}{e} d A_{(2)}^{(\mathrm{NS})} \wedge d A_{(2)}^{(\mathrm{R})} \wedge A_{(3)} .
\end{aligned}
$$

The reduction ansatz for the metric will be the usual one, namely

$$
d s_{9}^{2}=e^{2 \alpha f} d s_{5}^{2}+e^{2 \beta f} d s_{4}^{2}\left(C P^{2}\right)
$$

where, from appendix $\mathrm{A}$, the constants $\alpha$ and $\beta$ here are given by

$$
\alpha=\sqrt{\frac{2}{21}}, \quad \beta=-\frac{3}{4} \alpha .
$$

For the various field strengths, they will all just directly reduce naïvely, except for $F_{(4)}$ and $\mathcal{F}_{(2)}$, which will be reduced according to

$$
\begin{aligned}
& F_{(4)} \longrightarrow F_{(4)}+4 m \epsilon_{(4)}, \\
& \mathcal{F}_{(2)} \longrightarrow \mathcal{F}_{(2)}+2 \mu J,
\end{aligned}
$$

where $\epsilon_{(4)}$ is the volume-form on $C P^{2}$, and $J$ is the Kähler form.

We may now substitute the various ansätze into the nine-dimensional Lagrangian (2.38). To avoid repetitive formulae, let us also at this stage perform an $O(2)$ rotation of the scalars $(\varphi, f)$, to tilded ones:

$$
\begin{array}{llrl}
\tilde{f} & =\sqrt{\frac{3}{35}} f+4 \sqrt{\frac{2}{35}} \varphi, & \tilde{\varphi} & =4 \sqrt{\frac{2}{35}} f-\sqrt{\frac{3}{35}} \varphi, \\
f & =\sqrt{\frac{3}{35}} \tilde{f}+4 \sqrt{\frac{2}{35}} \tilde{\varphi}, & \varphi & =4 \sqrt{\frac{2}{35}} \tilde{f}-\sqrt{\frac{3}{35}} \tilde{\varphi} .
\end{array}
$$

In terms of these, the reduced five-dimensional Lagrangian turns out to be

$$
e^{-1} \mathcal{L}_{5}=R-\frac{1}{2}(\partial \phi)^{2}-\frac{1}{2}(\partial \tilde{\varphi})^{2}-\frac{1}{2}(\partial \tilde{f})^{2}-\frac{1}{2} e^{2 \phi}(\partial \chi)^{2}-\frac{1}{48} e^{-2 \sqrt{\frac{2}{5}} \tilde{f}-2 \sqrt{\frac{3}{5}}} \tilde{\varphi} F_{(4)}^{2}
$$




$$
\begin{aligned}
& -\frac{1}{12} e^{-\phi-\sqrt{\frac{5}{3}} \tilde{\varphi}}\left(F_{(3)}^{(\mathrm{NS})}\right)^{2}-\frac{1}{2} e^{\phi-\sqrt{\frac{5}{3}} \tilde{\varphi}}\left(F_{(3)}^{(\mathrm{R})}\right)^{2}-\frac{1}{4} e^{2 \sqrt{\frac{2}{5}} \tilde{f}-\frac{4}{\sqrt{15}} \tilde{\varphi}}\left(\mathcal{F}_{(2)}\right)^{2} \\
& -\frac{1}{4} e^{\phi-2 \sqrt{\frac{2}{5}} \tilde{f}-\frac{1}{\sqrt{15}} \tilde{\varphi}}\left(F_{(2)}^{(\mathrm{R})}\right)^{2}-\frac{1}{4} e^{-\phi-2 \sqrt{\frac{2}{5}} \tilde{f}-\frac{1}{\sqrt{15}} \tilde{\varphi}}\left(F_{(2)}^{(\mathrm{NS})}\right)^{2} \\
& -8 m^{2} e^{2 \sqrt{\frac{5}{3}} \tilde{\varphi}}-\mu^{2} e^{3 \sqrt{\frac{2}{5}} \tilde{f}+\frac{4}{\sqrt{15}} \tilde{\varphi}}+e^{\frac{1}{\sqrt{10}} \tilde{f}+\frac{4}{\sqrt{15}} \tilde{\varphi}} R_{4} \\
& -\frac{4}{e} m *\left(d A_{(3)} \wedge \mathcal{A}_{(1)}\right)-\frac{4}{e} m *\left(A_{(2)}^{(\mathrm{R})} \wedge d A_{(2)}^{(\mathrm{NS})}\right) .
\end{aligned}
$$

We can first perform the statutory consistency check of verifying that we can get back the results of the previous subsection, by truncating out the squashing mode. The rotated basis for the dilatons that we are using in (2.43) is adapted for the purpose, and indeed we can see it is consistent with the equation of motion for $\tilde{f}$ to set $\tilde{f}=0$, provided that $R_{4}=6 \mu^{2}$. If we then set $\tilde{f}=0$, we see that indeed the Lagrangian truncates to the previous one (2.34), with $\varphi$ replaced by $\tilde{\varphi}$.

It is also instructive to write the ansatz for the original ten-dimensional type IIB metric in terms of the tilded dilatons defined in $(2.42)$. From $(2.37)$ and $(2.39)$, we find that the ten-dimensional metric is given by

$$
d s_{10}^{2}=e^{\sqrt{\frac{5}{12}} \tilde{\varphi}} d s_{5}^{2}+e^{-\sqrt{\frac{5}{12}} \tilde{\varphi}}\left(e^{-\frac{1}{\sqrt{10}} \tilde{f}} d s_{4}^{2}\left(C P^{2}\right)+e^{\frac{4}{\sqrt{10}} \tilde{f}}\left(d z+\mathcal{A}_{(1)}\right)^{2}\right) .
$$

Thus indeed we see that $\tilde{f}$ acts as a volume-preserving "squashing mode" of the 5 -sphere, while $\tilde{\varphi}$ is the breathing mode.

\subsection{Further examples}

\subsubsection{Reduction of $D=6$ supergravity on $S^{3}$}

We may consider the reduction of a $D=6$ Lagrangian of the form

$$
e^{-1} \mathcal{L}_{6}=R-\frac{1}{2}(\partial \phi)^{2}-\frac{1}{12} e^{\sqrt{2} \phi} F_{(3)}^{2},
$$

where the internal space is taken to be $S^{3}$. This calculation is relevant for the reduction of any of the six-dimensional supergravities. Following the same procedures that we have used previously, we shall first consider the situation where an $S O(4)$-invariant reduction is performed, with the ansätze

$$
\begin{aligned}
& d \hat{s}_{6}^{2}=e^{2 \alpha \varphi} d s_{3}^{2}+e^{2 \beta \varphi} d s^{2}\left(S^{3}\right), \\
& \hat{F}_{(3)}=F_{(3)}+m \epsilon_{(3)}\left(S^{3}\right),
\end{aligned}
$$

where $\alpha=\sqrt{\frac{3}{8}}$ and $\beta=-\frac{1}{3} \alpha$. The resulting three-dimensional Lagrangian is

$$
e^{-1} \mathcal{L}_{3}=R-\frac{1}{2}(\partial \phi)^{2}-\frac{1}{2}(\partial \varphi)^{2}+R_{3} e^{\frac{8 \alpha}{3} \varphi}-\frac{1}{2} m^{2} e^{\sqrt{2} \phi+4 \alpha \varphi}-\frac{1}{12} e^{\sqrt{2} \phi-4 \alpha \varphi} F_{(3)}^{2} .
$$


We may now dualise $F_{(3)}$ in the standard way, solving its equation of motion by writing $F_{(3)}=c e^{-\sqrt{2} \phi+4 \alpha \varphi} \epsilon_{(3)}\left(S^{3}\right)$. The resulting dualised Lagrangian is

$$
e^{-1} \mathcal{L}_{3}=R-\frac{1}{2}(\partial \phi)^{2}-\frac{1}{2}(\partial \varphi)^{2}+R_{3} e^{\frac{8 \alpha}{3} \varphi}-\frac{1}{2} m^{2} e^{\sqrt{2} \phi+4 \alpha \varphi}-\frac{1}{2} c^{2} e^{-\sqrt{2} \phi+4 \alpha \varphi} .
$$

As in the previous examples, we may also consider a more general reduction where the squashing mode of the 3 -sphere is included, corresponding to the family of $S O(3) \times U(1)$ invariant metrics on the 3 -sphere described as a $U(1)$ bundle over $C P^{1}=S^{2}$. As before, this is most easily done by first reducing the six-dimensional theory on a circle, which will then be taken to be the $U(1)$ of the Hopf fibration. Thus our starting point now will be the five-dimensional theory obtained from (2.45) by reducing the metric according to

$$
d \hat{s}_{6}^{2}=e^{-\frac{1}{\sqrt{6} \varphi}} d s_{5}^{2}+e^{\frac{3}{\sqrt{6} \varphi}}\left(d z+\mathcal{A}_{(1)}\right)^{2},
$$

which gives the Lagrangian

$$
\begin{aligned}
e^{-1} \mathcal{L}_{5}= & R-\frac{1}{2}(\partial \phi)^{2}-\frac{1}{2}(\partial \varphi)^{2}-\frac{1}{12} e^{\sqrt{2} \phi+\frac{2}{\sqrt{6}} \varphi} F_{(3)}^{2} \\
& -\frac{1}{4} e^{\sqrt{2} \phi-\frac{2}{\sqrt{6}} \varphi} F_{(2)}^{2}-\frac{1}{4} e^{\frac{4}{\sqrt{6}} \varphi} \mathcal{F}_{(2)}^{2}
\end{aligned}
$$

We then perform a further reduction on $S^{2}$, with the ansätze

$$
\begin{aligned}
d s_{5}^{2} & \rightarrow e^{\frac{2}{\sqrt{3}} f} d s_{3}^{2}+e^{-\frac{1}{\sqrt{3}} f} d s^{2}\left(S^{2}\right), \\
F_{(3)} & \rightarrow F_{3}, \\
F_{(2)} & \rightarrow F_{(2)}+\lambda J, \\
\mathcal{F}_{(2)} & \rightarrow \mathcal{F}_{(2)}+m J .
\end{aligned}
$$

It is convenient also to make an orthogonal transformation on the basis for the dilatons $\varphi$ and $f$, defining

$$
f=\frac{2 \sqrt{2}}{3} \tilde{\varphi}+\frac{1}{3} \tilde{f}, \quad \varphi=-\frac{1}{3} \tilde{\varphi}+\frac{2 \sqrt{2}}{3} \tilde{f} .
$$

In this basis, and after dualising $F_{(3)}$ in $D=3$ by solving its equation of motion in the form $F_{(3)}=c e^{-\sqrt{2} \phi-\frac{2}{\sqrt{6}} \varphi+4 \alpha f}$, we obtain the three-dimensional Lagrangian

$$
\begin{aligned}
e^{-1} \mathcal{L}_{3}= & R-\frac{1}{2}(\partial \phi)^{2}-\frac{1}{2}(\partial \tilde{\varphi})^{2}-\frac{1}{2}(\partial \tilde{f})^{2}-\frac{1}{4} e^{\sqrt{2} \phi-\frac{2}{\sqrt{3}} \tilde{f}-\sqrt{\frac{2}{3}} \tilde{\varphi}} F_{(2)}^{2}-\frac{1}{4} e^{\frac{2}{\sqrt{3}} \tilde{f}-2 \sqrt{\frac{2}{3}} \tilde{\varphi}} \mathcal{F}_{(2)}^{2} \\
& -\frac{1}{2} \lambda^{2} e^{\sqrt{2} \phi+\sqrt{6} \tilde{\varphi}}-\frac{1}{2} c^{2} e^{-\sqrt{2} \phi+\sqrt{6} \tilde{\varphi}}-\frac{1}{2} m^{2} e^{\frac{4}{\sqrt{3}} \tilde{f}+2 \sqrt{\frac{2}{3}} \tilde{\varphi}}+R_{2} e^{\frac{1}{\sqrt{3}} \tilde{f}+2 \sqrt{\frac{2}{3}} \tilde{\varphi}} .
\end{aligned}
$$

As usual, we can verify that the equation of motion for $\tilde{f}$ can be satisfied by setting $\tilde{f}=0$, provided that $R_{2}=2 m^{2}$ and that $F_{(2)}=\mathcal{F}_{(2)}=0$. This corresponds to truncating the 
theory to the previous $S O(4)$-invariant reduction on $S^{3}$. The fact that $\tilde{f}$ is the volumepreserving squashing mode can be seen from the metric reduction ansatz, expressed in the tilded variables:

$$
d \hat{s}_{6}^{2}=e^{\sqrt{\frac{3}{2}} \tilde{\varphi}} d s_{3}^{2}+e^{-\frac{1}{\sqrt{6}} \tilde{\varphi}}\left(e^{-\frac{1}{\sqrt{3}} \tilde{f}} d s^{2}\left(S^{2}\right)+e^{\frac{2}{\sqrt{3}} \tilde{f}}\left(d z+\mathcal{A}_{(1)}\right)^{2}\right) .
$$

\subsubsection{Reduction of $D=5$ supergravity on $S^{2}$.}

To discuss this, it suffices to consider the subsector of the $D=5$ theory described by the Einstein-Maxwell Lagrangian

$$
e^{-1} \mathcal{L}_{5}=R-\frac{1}{4} \hat{F}_{(2)}^{2}
$$

We then reduce on $S^{2}$, with the ansatz

$$
d \hat{s}_{5}^{2}=e^{2 \alpha \varphi} d s_{3}^{2}+e^{2 \beta \varphi} d s^{2}\left(S^{2}\right), \quad \hat{F}_{(2)}=F_{(2)}+m \epsilon_{(2)}\left(S^{2}\right)
$$

where $\alpha=1 / \sqrt{3}$ and $\beta=-\alpha / 2$. The resulting three-dimensional Lagrangian turns out to be

$$
e^{-1} \mathcal{L}_{3}=R-\frac{1}{2}(\partial \varphi)^{2}-\frac{1}{4} e^{-2 \alpha \varphi} F_{(2)}^{2}-\frac{1}{2} m^{2} e^{4 \alpha \varphi}+R_{2} e^{3 \alpha \varphi} .
$$

\section{Supersymmetric domain walls}

\subsection{Domain wall in $D=4$}

The Lagrangian (2.3) of the $S O(8)$-singlets in the $S^{7}$ reduction admits a supersymmetric domain wall (i.e. membrane) solution in $D=4$. For convenience, we shall discuss the solutions using the Lagrangian (2.6), where the $F_{4}$ term has been dualised into a cosmological term. Comparing this with the general Lagrangian $(\overline{B .1}, \bar{B} .2)$ in appendix $B$, we see that $a_{1}=6 \alpha, a_{2}=\frac{18}{7} \alpha, g_{1}=c, g_{2}=\sqrt{2 R_{7}}$, and $\lambda=0$, which is consistent with the requirement (B.6). The solution is therefore given by $\tilde{b}_{1}= \pm 5 c /(2 k), \tilde{b}_{2}= \pm 5 \sqrt{6 R_{7} / 7} /(2 k)$, $\nu_{1}=\frac{1}{2}\left(a_{2}-a_{1}\right)=\frac{5}{\sqrt{7}}$ and $\nu_{2}=6$, and hence

$$
\begin{aligned}
e^{-\frac{5}{\sqrt{7}} \varphi} & =H=e^{-\frac{5}{\sqrt{7}} \varphi_{0}}+k|y|, \\
e^{3 A} & =e^{-B}=\tilde{b}_{1} H^{\frac{3}{10}}+\tilde{b}_{2} H^{\frac{7}{10}},
\end{aligned}
$$

with the metric of the form given by $(\overline{\mathrm{B} .3})$ :

$$
d s_{4}^{2}=\left(\tilde{b}_{1} H^{\frac{3}{10}}+\tilde{b}_{2} H^{\frac{7}{10}}\right)^{\frac{2}{3}} d x^{\mu} d x_{\mu}+\left(\tilde{b}_{1} H^{\frac{3}{10}}+\tilde{b}_{2} H^{\frac{7}{10}}\right)^{-2} d y^{2}
$$


Note that here we have four different solutions, depending on the signs of $\tilde{b}_{i}$. We shall show presently that these solutions preserve one half of the supersymmetry. We shall consider the case where these two parameters are of opposite sign, in order to have solutions that are real. In this case, when $k \rightarrow 0$, the solution reduces to $\mathrm{AdS}_{4}$, with $e^{\frac{24 \alpha}{7} \varphi}=e^{\frac{24 \alpha}{7} \varphi_{\text {AdS }}}$ (see appendix B for a general derivation of domain-wall solutions).

We shall first consider the case where the constant $\varphi_{0}$ in the solution (3.1) is given by $\varphi_{0}=\varphi_{\text {AdS }}$, implying that the geometry near $y=0$ approaches $\mathrm{AdS}_{4}$. To see this, we note that in the region $|y| \rightarrow 0$, the metric can be written as $d s^{2} \sim e^{2 \rho / 3} d x^{\mu} d x_{\mu}+d \rho^{2}$, with $\rho=\log |y| \rightarrow-\infty$. This is $\mathrm{AdS}_{4}$, written in horospherical coordinates. Note that if $k$ is positive, then the solution is real for all values of $y$, provided that $\tilde{b}_{2}>0$ and $\tilde{b}_{1}<0$. In this case we can take $y$ to run from $-\infty$ to $\infty$. In the regions where $|y| \rightarrow \infty$ the $H^{\frac{7}{10}}$ term in (3.1) dominates. The curvature tends to zero in these regions, so the metric is asymptotically locally flat, and its behaviour is dominated by the contribution from the $R_{7}$ potential term (i.e. the $H^{\frac{7}{10}}$ term), while the dilaton approaches negative infinity in this asymptotic limit. The solution is reflection-symmetric about $y=0$, where the solution approaches $\mathrm{AdS}_{4}$. It deviates more and more from $\mathrm{AdS}_{4}$ as $|y|$ increases, with the curvature eventually vanishing at $|y|=\infty$. The $y=0$ point is a horizon, since $g_{00}=0$ there. If $k$ is taken to be negative instead, then the solution is real for $|y|<y_{0} \equiv e^{-\frac{5}{\sqrt{7}} \varphi_{\text {Ads }}} /|k|$. In the regions $|y| \rightarrow y_{0}$, where $H$ vanishes, the curvature becomes singular and its behaviour is then dominated by the $c^{2}$ potential term (i.e. the metric is dominated by the term $H^{\frac{3}{10}}$ in (3.1).) The dilaton approaches positive infinity in this limit. Thus, in this $k<0$ case, the solution is again reflection-symmetric, and approaches $\mathrm{AdS}_{4}$ at $y=0$, but it now has curvature singularities at $y= \pm y_{0}$. In both the positive and negative $k$ cases, the metric functions $A$ and $B$ can be expressed as

$$
e^{3 A}=e^{-B}=\frac{5}{2 k}\left(\sqrt{6 R_{7} / 7} H^{\frac{7}{10}}-c H^{\frac{3}{10}}\right),
$$

corresponding to $b_{1}<0$ and $b_{2}>0$, where $b_{i}$ is defined in (B.5).

Different situations can arise if the constant $\varphi_{0}$ takes values other than $\varphi_{\text {Ads }}$. In the case $k>0$, for which $y$ runs from $-\infty$ to $\infty$, then if $\varphi_{0}>\varphi_{A d S}$ the metric reaches the AdS form at the points $y_{ \pm}= \pm k^{-1}\left(e^{-5 \varphi_{A d S} / \sqrt{7}}-e^{-5 \varphi_{0} / \sqrt{7}}\right)$. There is a domain wall at $y=0$, with a delta-function curvature singularity. Thus the domain wall divides the spacetime into two mirror-symmetric regions which each has an $\mathrm{AdS}_{4}$ as its near-horizon structure. The domain wall is inside the horizon. If, on the other hand $\varphi_{0}<\varphi_{A d S}$ then $g_{00}$ never reaches zero, and the domain wall at $y=0$ divides the spacetime into two regions that never 
reach the $\mathrm{AdS}_{4}$ form. The situation is different if $k<0$, in that now, instead of $y$ running inwards from a flat region at $|y|=\infty$, it runs from curvature singularities at $y= \pm y_{0}$. Again, depending on whether $\varphi_{0}$ is greater than or less than $\varphi_{A d S}$, the metric either passes through the $\mathrm{AdS}_{4}$ region before reaching the domain wall, or else it reaches the domain wall without encountering an $\mathrm{AdS}_{4}$ region. Note that the delta-function curvature singularity at $y=0$, which arises because the harmonic function is taken to depend on $y$ through its modulus $|y|$, is absent in the special case $\varphi_{0}=\varphi_{A d S}$ described in the previous paragraph, but is otherwise generically present. (For a review of domain walls in $D=4$ supergravities, see [28].)

Although it might seem a bizarre choice from the $D=4$ perspective, another patchingtogether of segments of the solution (3.1) is possible. One can match the $k<0$ solution for $y<0$ onto the $k>0$ solution for $y>0$. This effectively removes the absolute value prescription for $y$ in $H$, thus removing also the delta-function curvature singularity at $y=0$. Although the metric (3.1) apparently becomes singular at $y=0$, this proves to be just a coordinate singularity, and the spacetime has in fact a regular horizon there. The price to be paid for this choice of patching is the appearance of a genuine curvature singularity at $y=-y_{0}$, which can be reached by a lightlike or timelike geodesic at a finite affineparameter value. From a $D=4$ viewpoint, the natural choice would seem to be the first one made above: taking $H$ to depend on $|y|$ with $k>0$, and tolerating a rather mild deltafunction singularity at $y=0$. We shall shortly see, however, that the "patched" solution in fact corresponds directly to the most natural metric from the $D=11$ point of view. We shall return in the next subsection to a more general study of the spherical oxidations of domain-wall solutions.

The general solution can be straightforwardly oxidised back to $D=11$, giving

$$
\begin{aligned}
d s_{11}^{2} & =e^{2 \alpha \varphi+2 A} d x^{\mu} d x_{\mu}+e^{2 \alpha \varphi-6 A} d y^{2}+e^{2 \beta \varphi} d s_{7}^{2} \\
& =\left(\tilde{b}_{1} H^{-\frac{2}{5}}+\tilde{b}_{2}\right)^{\frac{2}{3}} d x^{\mu} d x^{\nu} \eta_{\mu \nu}+\left(\tilde{b}_{1} H^{\frac{8}{15}}+\tilde{b}_{2} H^{\frac{14}{15}}\right)^{-2} d y^{2}+H^{\frac{2}{15}} d s_{7}^{2} \\
F_{4} & =c e^{6 \alpha \varphi} \epsilon_{4}=c H^{-\frac{7}{5}} \epsilon_{4}=c H^{-\frac{7}{5}} d^{3} x \wedge d y
\end{aligned}
$$

From the eleven-dimensional point of view, the solution has a total of four free parameters, namely $\varphi_{0}, k, c$ and $R_{7}$. There are three special limits, depending on the values of these parameters. As we have seen, when $k=0$ and $e^{\frac{24 \alpha}{7} \varphi}=6 R_{7} /\left(7 c^{2}\right)$, the solution is $\operatorname{AdS}_{4} \times S^{7}$. If $c=0$, the metric is in fact purely Minkowskian. If $R_{7}=0, e . g$. when $d s_{7}^{2}$ is a flat metric, then the solution becomes a membrane with its charges uniformly distributed over the seven-dimensional surface 34. 
It is now fairly simple to check the supersymmetry of the domain-wall solution. This is most easily done by looking at the solution in eleven dimensions, as given in (3.4). First, we note that a metric of the form

$$
d \hat{s}^{2}=e^{2 f} d x^{\mu} d x_{\mu}+e^{2 h} d y^{2}+e^{2 u} d s^{2}
$$

where $f, h$ and $u$ depend only on $y$, has a spin connection, in the natural vielbein basis $\hat{e}^{\mu}=e^{f} d x^{\mu}, \hat{e}^{y}=e^{h} d y, \hat{e}^{a}=e^{u} e^{a}$, given by

$$
\hat{\omega}^{\mu y}=f^{\prime} e^{f-h} d x^{\mu}, \quad \hat{\omega}^{a y}=u^{\prime} e^{u-h} e^{a}, \quad \hat{\omega}^{a b}=\omega^{a b} .
$$

In the present case we have $f=\alpha \varphi+A, h=\alpha \varphi-3 A$, and $u=\beta \varphi$. Substituting into the $D=11$ supersymmetry transformation rule 29.

$$
\delta \psi_{M}=D_{M} \epsilon-\frac{1}{288}\left(\Gamma_{M}^{N_{1} \cdots N_{4}} F_{N_{1} \cdots N_{4}}-8 \Gamma^{N_{1} N_{2} N_{3}} F_{M N_{1} N_{2} N_{3}}\right) \epsilon,
$$

we find that the condition $\delta \psi_{\mu}=0$ gives

$$
\delta \psi_{\mu}=\partial_{\mu} \epsilon-\frac{1}{2} e^{f-h} \Gamma_{y}\left(f^{\prime} \Gamma_{\mu}-\frac{1}{6} m e^{3 \alpha \varphi-3 A} \varepsilon_{\mu \nu \rho} \Gamma^{\nu \rho}\right) \epsilon=0
$$

implying that for preserved supersymmetry we must have

$$
f^{\prime}=\alpha \varphi^{\prime}+A^{\prime}=-\frac{1}{3} m e^{3 \alpha \varphi-3 A} .
$$

(Actually, we have the freedom here to choose either a plus or a minus sign on the right-hand side; we choose the minus sign in order to agree with the choice made previously, where $b_{1}$ was taken to be negative.) Similarly, from the condition that $\delta \psi_{a}=0$, we obtain another first-order equation, this time for $\varphi$ alone, namely

$$
\delta \psi_{a}=D_{a} \epsilon+\frac{1}{2} e^{u-h} \Gamma_{a y}\left(u^{\prime}-\frac{1}{6} m e^{3 \alpha \varphi-3 A} \Gamma_{012}\right) \epsilon=0
$$

Note that the explicit indices on $\Gamma_{012}$ here refer to the vielbein directions in the space of the $x^{\mu}$ coordinates. From this, we see that the existence of Killing spinors will require that

$$
u^{\prime}=-\frac{2}{7} \alpha \varphi^{\prime}=\frac{1}{6} m e^{3 \alpha \varphi-3 A}-\kappa e^{\frac{9}{7} \alpha \varphi-3 A},
$$

since then we will have

$$
\delta \psi_{a}=\left(D_{a}-\frac{1}{2} \kappa \Gamma_{a y}\right) \epsilon-\frac{1}{12} m e^{\frac{12 \alpha}{7} \varphi} \Gamma_{a y}\left(1-\Gamma_{012}\right) \epsilon=0
$$

This will have solutions provided that the constant $\kappa$ is given by $\kappa=\sqrt{\frac{R_{7}}{42}}$, since then the first expression in the brackets is the $S^{7}$-covariant derivative encountered previously in 
the Kaluza-Klein reduction of $D=11$ supergravity on the 7-sphere [30]. In fact, the two first-order conditions that we have just obtained are precisely the ones given in (B.5) in appendix B, where the equations of motion for domain walls are solved. (Note that we have $b_{1}=-\sqrt{7} m / 2<0$ and $b_{2}=\sqrt{\frac{3}{2} R_{7}}>0$ here.) Thus the conditions above reduce to

$$
\begin{aligned}
\delta \psi_{\mu} & =\partial_{\mu} \epsilon+\frac{1}{6} m e^{3 \alpha \varphi+A} \Gamma_{y}\left(\Gamma_{\mu}+\frac{1}{2} \varepsilon_{\mu \nu \rho} \Gamma^{\nu \rho}\right) \epsilon=0 \\
\delta \psi_{a} & =\left(D_{a}-\frac{1}{2} \sqrt{\frac{R_{7}}{42}} \Gamma_{a y}\right) \epsilon+\frac{1}{12} m e^{\frac{12 \alpha}{7} \varphi} \Gamma_{a y}\left(1-\Gamma_{012}\right) \epsilon=0 \\
\delta \psi_{y} & =\partial_{y} \epsilon+\frac{1}{6} m e^{3 \alpha \varphi-3 A} \Gamma_{012} \epsilon=0 .
\end{aligned}
$$

In all of the above expressions, $a$ is a vielbein index in the metric $d s_{7}^{2}$, all indices on Dirac matrices are vielbein indices, the indices $\mu$ and $y$ on $\psi$ and $\partial$ are world indices, and $\varepsilon_{\mu \nu \rho}$ is the tensor density taking the values $\pm 1,0$. It is now easily seen that $\epsilon$ will be a Killing spinor if it satisfies the conditions

$$
\epsilon=e^{\frac{1}{2} f} \epsilon_{0} \otimes \eta=e^{\frac{1}{2}(\alpha \varphi+A)} \epsilon_{0} \otimes \eta, \quad \Gamma_{012} \epsilon_{0}=\epsilon_{0}
$$

where $\epsilon_{0}$ is a constant spinor in the four-dimensional spacetime, and $\eta$ is a Killing spinor in the internal seven-dimensional space, satisfying

$$
D_{a} \eta-\frac{i}{2} \sqrt{\frac{R_{7}}{42}} \gamma_{a} \eta=0
$$

We are assuming here that the eleven-dimensional Dirac matrices are decomposed as $\Gamma_{\mu}=$ $\gamma_{\mu} \otimes \mathbb{1}, \Gamma_{y}=\gamma_{y} \otimes \mathbb{1}$, and $\Gamma_{a}=\gamma_{5} \otimes \gamma_{a}$, with $\gamma_{5}=i \gamma_{012 y}$. In the case where the internal space is $S^{7}$, there will be eight Killing spinors $\eta$ satisfying (3.15), and so we see that the domain-wall solution, and its oxidation to $D=11$, preserves one half of the supersymmetry.

Having seen that the eleven-dimensional metric (3.4), obtained by oxidising the fourdimensional domain-wall solution, preserves half of the supersymmetry, we now observe that it can in fact be re-interpreted as the standard BPS membrane solution of $D=11$ supergravity. To see this, let us introduce a new coordinate $\rho$, related to the coordinate $y$ in (3.4) by

$$
\rho=\sqrt{\frac{42}{R_{7}}} H^{\frac{1}{15}}=\sqrt{\frac{42}{R_{7}}}\left(e^{-\frac{5}{\sqrt{7}} \varphi_{0}}+k y\right)^{\frac{1}{15}} .
$$

It is then easy to see that (3.4) becomes, upon substituting (3.16) for $y>0$ and then continuing the result in $\rho$ so as to cover the full range $0<\rho<\infty$,

$$
d s_{11}^{2}=\tilde{b}_{2}^{\frac{2}{3}}\left(1-\frac{\tilde{k}}{\rho^{6}}\right)^{\frac{2}{3}} d x^{\mu} d x_{\mu}+\left(1-\frac{\tilde{k}}{\rho^{6}}\right)^{-2} d \rho^{2}+\rho^{2} d \Omega_{7}^{2},
$$


where $\tilde{k}=\frac{1}{6} c\left(42 / R_{7}\right)^{7 / 2}$, and $d \Omega_{7}^{2}$ is the metric on the unit 7-sphere. This can be recognised as the standard form for the $D=11$ BPS membrane solution, written in Schwarzschild type coordinates.

At this point, we can make contact with the "patched" $D=4$ domain-wall solution mentioned above, which joins together a $k>0$ solution for $y>0$ with a $-k$ solution for $y<0$. Although this seems unnatural from the $D=4$ viewpoint, what one obtains after oxidation up to $D=11$ is indeed a natural variant of the $D=11$ membrane solution. In this solution, $y$ is viewed as a radial coordinate for a non-isotropic coordinate system, hence naturally bounded to take values on a half-line. This solution is in fact the maximal analytic extension [31] of the $D=11$ membrane solution [32], with a non-singular horizon at $y=0$ and a central timelike core singularity at $y=-y_{0}$.

\subsection{Oxidation of domain walls}

In the previous subsection, we saw that the domain-wall solution of the $N=8$ fourdimensional supergravity obtained by reducing $D=11$ supergravity on $S^{7}$ admits a simple interpretation after oxidation back to $D=11$, namely as the standard membrane solution. Now, we shall show that in fact this is a rather general feature of the domain-wall solutions of supergravities reduced on spheres, and in fact they can all be re-interpreted as extremal $p$-branes back in their original higher-dimensional theories.

To see this, we begin by noting that the general result (A.13) for the dimensional reduction of gravity plus an antisymmetric-tensor field strength of degree $d_{x}$ or $d_{y}$ from $D=d_{x}+d_{y}$ dimensions to $d_{x}$ dimensions is precisely of the form that we consider in appendix B, which allowed us to obtain domain-wall solutions. Specifically, in the notation of appendix $\mathrm{B}$, we have

$$
g_{1}^{2}=m^{2}, \quad g_{2}^{2}=2 R_{y}, \quad a_{1}=2 \alpha\left(d_{x}-1\right), \quad a_{2}=2(\alpha-\beta) .
$$

It is easily verified that $a_{1}$ and $a_{2}$ are such that the condition (B.7) is satisfied, implying that indeed we have domain-wall solutions with $\lambda$ in (B.6) vanishing. In terms of the parameters $\tilde{b}_{1}, \tilde{b}_{2}$ defined in appendix $\mathrm{B}$, the domain-wall solutions are therefore given by

$$
\begin{aligned}
e^{2 \alpha \varphi} & =H^{-2 d_{y} /\left(d_{x} d_{y}+d_{x}-2\right)}, \\
e^{\left(d_{x}-1\right) A} & =e^{-B}=\tilde{b}_{1} H^{\left(d_{x}+d_{y}-2\right) /\left(d_{x} d_{y}+d_{x}-2\right)}+\tilde{b}_{2} H^{\left(d_{x}-1\right) d_{y} /\left(d_{x} d_{y}+d_{x}-2\right)} .
\end{aligned}
$$

Oxidising back to $D$ dimensions, using the formulae given in appendix $\mathrm{A}$, we find that the 
metric becomes

$$
\begin{aligned}
d s_{D}^{2}= & \left(\tilde{b}_{2}+\tilde{b}_{1} H^{-\left(d_{x}-2\right)\left(d_{y}-1\right) /\left(d_{x} d_{y}+d_{x}-2\right)}\right)^{2 /\left(d_{x}-1\right)} d x^{\mu} d x_{\mu} \\
& +\left(\tilde{b}_{2} H^{d_{x} d_{y} /\left(d_{x} d_{y}+d_{x}-2\right)}+\tilde{b}_{1} H^{\left(d_{x}+2 d_{y}-2\right) /\left(d_{x} d_{y}+d_{x}-2\right)}\right)^{-2} d y^{2} \\
& +\lambda^{-2} H^{2\left(d_{x}-2\right) /\left(d_{x} d_{y}+d_{x}-2\right)} d \Omega_{d_{y}}^{2},
\end{aligned}
$$

where $\lambda^{2}=R_{y} /\left(d_{y}\left(d_{x}-1\right)\right)$.

As in the previous subsection, we may now introduce a new coordinate $\rho$ in place of $y$, defined here by

$$
\rho=\lambda^{-1} H^{\left(d_{x}-2\right) /\left(d_{x} d_{y}+d_{x}-2\right)} .
$$

In terms of $r$, the oxidised domain-wall metric (3.20) becomes (recalling that $\tilde{b}_{1}<0, \tilde{b}_{2}>0$ ).

$$
d s_{D}^{2}=\tilde{b}_{2}^{2 /\left(d_{x}-1\right)}\left(1-\frac{\tilde{k}}{\rho^{d_{y}-1}}\right)^{2 /\left(d_{x}-1\right)} d x^{\mu} d x_{\mu}+\left(1-\frac{\tilde{k}}{\rho^{d_{y}-1}}\right)^{-2} d \rho^{2}+\rho^{2} d \Omega_{d_{y}}^{2},
$$

where $\tilde{k}=\lambda^{-d_{y}+1}\left|\tilde{b}_{1} / \tilde{b}_{2}\right|$. Finally, we make the replacement $x^{\mu} \rightarrow \tilde{b}_{2}^{-1 /\left(d_{x}-1\right)} x^{\mu}$, and define a new radial coordinate $r$ by $\rho^{d_{y}-1}=r^{d_{y}-1}+\tilde{k}$, in terms of which the metric becomes

$$
d s^{2}=\left(1+\frac{\tilde{k}}{r^{d_{y}-1}}\right)^{-2 /\left(d_{x}-1\right)} d x^{\mu} d x_{\mu}+\left(1+\frac{\tilde{k}}{r^{d_{y}-1}}\right)^{2 /\left(d_{y}-1\right)}\left(d r^{2}+r^{2} d \Omega_{d_{y}}^{2}\right) .
$$

This can be recognised as the standard form of an isotropic non-dilatonic extremal $p$-brane with $p=d_{x}-2$. In the context of supergravity theories, it therefore follows that the domain-wall solutions that we are considering in this paper all preserve one half of the supersymmetry. Indeed, in the previous subsection where we considered the example of the domain wall in $D=4$, we gave an explicit derivation of this result.

\subsection{Further examples}

It is straightforward to see that the two-parameter supersymmetric domain-wall solution obtained in section 3.1 can be embedded in the Lagrangian (2.20) including the squashing mode, since (2.20) reduces to the Lagrangian (2.3) when the dilaton $\tilde{\phi}$ is consistently set to zero.

The $D=7$ Lagrangian (2.23) fits the pattern of (B.1) and (B.2), with $a_{1}=12 \alpha$ and $a_{2}=\frac{9}{2} \alpha$. It follows from (B.6) that $\lambda=0$. The two-parameter domain-wall solution is given by

$$
\begin{aligned}
d s_{7}^{2} & =e^{2 A} d x^{\mu} d x^{\nu} \eta_{\mu \nu}+e^{2 B} d y^{2}, \\
e^{-\frac{11}{2 \sqrt{10}} \varphi} & =H=e^{-\frac{11}{2 \sqrt{10}} \varphi_{0}}+k|y|, \quad B=-6 A \\
e^{6 A} & =\tilde{b}_{1} H^{\frac{3}{11}}+\tilde{b}_{2} H^{\frac{8}{11}}
\end{aligned}
$$


where $\tilde{b}_{1}= \pm 66 m /(5 k)$ and $\tilde{b}_{2}= \pm(11 / 10 k) \sqrt{3 R_{4}}$. If we oxidise the solution back to $D=11$, we have

$$
\begin{aligned}
d s_{11}^{2} & =e^{2 \alpha \varphi+2 A} d x^{\mu} d x_{\mu}+e^{2 \alpha \varphi-12 A} d y^{2}+e^{2 \beta \varphi} d s_{4}^{2}\left(S^{4}\right) \\
& =\left(\tilde{b}_{1} H^{-\frac{5}{11}}+\tilde{b}_{2}\right)^{\frac{1}{3}} d x^{\mu} d x^{\nu} \eta_{\mu \nu}+\left(\tilde{b}_{1} H^{\frac{13}{33}}+\tilde{b}_{2} H^{\frac{28}{33}}\right)^{-2} d y^{2}+H^{\frac{10}{33}} d s_{4}^{2} \\
F_{4} & =6 m \epsilon_{4} .
\end{aligned}
$$

The $\mathrm{AdS}_{7} \times S^{4}$ solution arises in the limit $k \rightarrow 0$ when $\tilde{b}_{1}$ and $\tilde{b}_{2}$ have opposite signs, and $e^{5 \varphi_{0} / \sqrt{10}}=R_{4} /\left(48 m^{2}\right)$. When $m=0$, the spacetime is flat, whilst if $R_{4}=0$, the solution describes a 5-brane with its charges uniformly distributed over $d s_{4}^{2}$ [34]. From the general discussion in section 3.2, we can easily see that this eleven-dimensional solution is nothing but the standard extremal 5-brane. This implies in particular that the $D=7$ domain-wall solution preserves half the supersymmetry.

Another example of a domain-wall solution arises in $D=5$, in the theory obtained by reducing type IIB supergravity on $S^{5}$. There is in this case a two-parameter domain-wall solution given by

$$
\begin{aligned}
d s_{5}^{2} & =e^{2 A} d x^{\mu} d x^{\nu} \eta_{\mu \nu}+e^{2 B} d y^{2}, \\
e^{-\frac{7}{\sqrt{15}} \varphi} & =H=e^{-\frac{7}{\sqrt{3}} \varphi_{0}}+k|y|, \quad B=-4 A \\
e^{4 A} & =\tilde{b}_{1} H^{\frac{2}{7}}+\tilde{b}_{2} H^{\frac{5}{7}},
\end{aligned}
$$

where $\tilde{b}_{1}= \pm 28 m /(3 k)$ and $\tilde{b}_{2}= \pm 14 /(15 k) \sqrt{5 R_{5}}$. This can be oxidised back to $D=10$, where it becomes

$$
\begin{aligned}
d s_{10 \mathrm{IIB}}^{2} & =e^{2 \alpha \varphi+2 A} d x^{\mu} d x_{\mu}+e^{2 \alpha \varphi-8 A} d y^{2}+e^{2 \beta \varphi} d s^{2}\left(S^{5}\right) \\
& =\left(\tilde{b}_{1} H^{-\frac{3}{7}}+\tilde{b}_{2}\right)^{\frac{1}{2}} d x^{\mu} d x^{\nu} \eta_{\mu \nu}+\left(\tilde{b}_{1} H^{\frac{13}{28}}+\tilde{b}_{2} H^{\frac{25}{28}}\right)^{-2} d y^{2}+H^{\frac{3}{14}} d s^{2}\left(S^{5}\right) \\
H_{(5)} & =4 m e^{8 \alpha \varphi} \epsilon_{(5)}+4 m \epsilon_{(5)}\left(S^{5}\right) \\
& =4 m e^{8 \alpha \varphi} d^{4} x \wedge d y+4 m \epsilon_{(5)}\left(S^{5}\right) .
\end{aligned}
$$

The AdS structure arises when $\tilde{b}_{1}$ and $\tilde{b}_{2}$ have opposite signs, with $k=0$ and $e^{2 \sqrt{\frac{3}{5}} \varphi_{0}}=$ $R_{5} /\left(20 m^{2}\right)$. If $m=0$, the solution is flat spacetime; if $R_{5}=0$, the solution is a D3brane with its charges uniformly distributed on $d s_{5}^{2}$. From the results given in section 3.2, the metric (3.27) can be seen to be equivalent to that of the standard extremal self-dual 3-brane of type IIB supergravity. Again, a consequence is that the $D=5$ domain-wall solution preserves one half of the supersymmetry. 
Another example of a domain-wall solution arises for the three-dimensional theory (2.48), obtained by compactifying six-dimensional supergravity on $S^{3}$. It is easily seen that this admits an $\mathrm{AdS}_{3}$ solution where $\phi$ and $\varphi$ are constants, given by

$$
e^{\sqrt{2} \phi_{\mathrm{AdS}}}=\frac{c}{m}, \quad e^{\frac{4 \alpha}{3} \varphi_{\mathrm{AdS}}}=\frac{2 R_{3}}{3 c m}
$$

We may now consistently truncate out the dilaton $\phi$, i.e. by setting $\phi=\phi_{\text {AdS }}$, so that the Lagrangian (2.48) then becomes

$$
e^{-1} \mathcal{L}=R-\frac{1}{2}(\partial \varphi)^{2}-m c e^{4 \alpha \varphi}+R_{3} e^{\frac{8 \alpha}{3} \varphi}
$$

which fits the pattern of the Lagrangian (B.1) and (B.2) with $a_{1}=4 \alpha, a_{2}=8 \alpha / 3$, and $\lambda=0$. Thus this Lagrangian admits a domain-wall (i.e. string) solution, given by

$$
\begin{aligned}
d s_{3}^{2} & =e^{2 A} d x^{\mu} d x_{\mu}+e^{2 B} d y^{2}, \quad e^{-\frac{5}{\sqrt{6}} \varphi}=H \equiv e^{-\frac{5}{\sqrt{6}} \varphi_{\text {AdS }}}+k|y|, \\
e^{2 A} & =e^{-B}=\tilde{b}_{1} H^{\frac{2}{5}}+\tilde{b}_{2} H^{\frac{3}{5}}
\end{aligned}
$$

where $\tilde{b}_{1}= \pm(5 / k) \sqrt{m c}$ and $\tilde{b}_{2}= \pm(5 / k) \sqrt{2 R_{3} / 3}$.

Finally, we consider the three-dimensional Lagrangian (2.57), obtained by reducing $D=$ 5 supergravity on $S^{2}$. This Lagrangian fits the pattern of (B.1) and (B.2) with $a_{1}=4 \alpha$, $a_{2}=3 \alpha$ and $\lambda=0$, so it admits a domain-wall solution

$$
\begin{aligned}
d s_{3}^{2} & =e^{2 A} d x^{\mu} d x_{\mu}+e^{2 B} d y^{2}, \quad e^{-\frac{7}{2 \sqrt{3}} \varphi}=H \equiv e^{-\frac{7}{2 \sqrt{3}} \varphi_{\text {AdS }}}+k|y|, \\
e^{2 A} & =e^{-B}=\tilde{b}_{1} H^{\frac{3}{7}}+\tilde{b}_{2} H^{\frac{4}{7}}
\end{aligned}
$$

where $\tilde{b}_{1}= \pm 7 m /(\sqrt{3} k)$ and $\tilde{b}_{2}= \pm(7 / k) \sqrt{R_{2} / 2}$

\section{Cosmological instanton solutions}

The Lagrangians that we obtained in section 2 by considering the dimensional reductions of supergravities on spheres have structures that are very similar to those considered in the context of cosmological instanton solutions in [1, 仙. In the simplest cases, for example, where we retain just the metric and the breathing-mode scalar as dynamical fields, we obtain Lagrangians of the form $\mathcal{L}=e R-\frac{1}{2} e(\partial \varphi)^{2}-e V(\varphi)$. In this section, we shall examine the cosmological instanton solutions in the case of the four-dimensional theory obtained in section 2.1 by making an $S O(8)$-invariant truncation of the reduction of $D=11$ supergravity on $S^{7}$. 
We begin by considering an $S O(4)$-invariant metric ansatz, in the co-moving frame, given by

$$
d s^{2}=-d \tau^{2}+b(\tau)^{2} d \Omega_{3}^{2}
$$

where $\tau$ is the co-moving time coordinate and $d \Omega_{3}^{2}$ is the metric on the unit 3 -sphere. It follows from (2.4) and the formulae in appendix $\mathbb{C}$ that the equations of motion are given by

$$
\ddot{\varphi}+3 \dot{\varphi} \frac{\dot{b}}{b}=-V_{, \varphi}(\varphi), \quad \ddot{b}=-\frac{1}{6} b\left(\dot{\varphi}^{2}-V(\varphi)\right)
$$

together with the first-order constraint

$$
\left(\frac{\dot{b}}{b}\right)^{2}+\frac{1}{b^{2}}=\frac{1}{12} \dot{\varphi}^{2}+\frac{1}{6} V,
$$

where $V$ is the scalar potential, given by (2.7). Note that the potential has the properties

$$
\begin{aligned}
V\left(\varphi_{0}\right) & =0, \quad \text { when } \quad e^{\frac{24 \alpha}{7} \varphi_{0}}=\frac{2 R_{7}}{c^{2}}, \\
V_{, \varphi}\left(\varphi_{0}\right) & =\frac{24 \alpha}{7} e^{\frac{18 \alpha}{7} \varphi_{0}} R_{7}=\frac{24 \alpha}{7} R_{7}\left(\frac{2 R_{7}}{c^{2}}\right)^{\frac{3}{4}}>0 .
\end{aligned}
$$

Thus we see that the contribution from $F_{4}=c e^{6 \alpha \varphi} \epsilon_{4}$ plays an important role in allowing the vanishing of the potential $V$ at a non-singular value $\varphi=\varphi_{0}$ of the dilaton. The potential also has a minimum at $\varphi=\varphi_{\text {AdS }}$ :

$$
\begin{aligned}
V_{, \varphi}\left(\varphi_{\mathrm{AdS}}\right) & =0, \quad \text { when } \quad e^{\frac{24 \alpha}{7} \varphi_{\mathrm{AdS}}}=\frac{6 R_{7}}{7 c^{2}}, \\
V_{\min } & =V\left(\varphi_{\mathrm{AdS}}\right)=-\frac{4}{7} R_{7} e^{\frac{18 \alpha}{7} \varphi_{\mathrm{AdS}}}=-\frac{4}{7} R_{7}\left(\frac{6 R_{7}}{7 m^{2}}\right)^{\frac{3}{4}}<0 .
\end{aligned}
$$

Here we are denoting by $\varphi_{\text {AdS }}$ the value of the dilaton for which the potential is a minimum. This is because the Lagrangian can be further truncated to

$$
e^{-1} \mathcal{L}=R-V_{\min }
$$

when $\varphi=\varphi_{\text {AdS }}$, and the equations of motion following from this Lagrangian allow an AdS solution. Note that we have $\varphi_{\text {AdS }}<\varphi_{0}$. Thus we see that this potential has a shape like a scoop. At large but negative $\varphi$. we have $V(\varphi) \rightarrow 0_{-}$, reducing as $\varphi$ increases, until it reaches $V_{\min }$ at $\varphi=\varphi_{\text {AdS }}$. The potential then increases monotonically to infinity, behaving like a single exponential for large $\varphi$.

The equations of motion (4.2,4.3) are invariant under time translations, and hence we can without loss of generality choose our initial time to be $\tau=0$. Following [1], we assume 
that at $\tau=0, \dot{\varphi}(0)=0$ and $V(\varphi(0)) \geq 0$, and hence $\varphi(0) \geq \varphi_{0}$. It then follows from the constraint (4.3) that at early times the solution for the metric function $b$ is imaginary 3

$$
b=i \tau
$$

and so the solution is more naturally discussed in a Euclideanised framework. In fact in the vicinity of $\tau=0$ the metric describes a Euclidean flat space.

We shall in fact present results for two different Euclidean-signature theories. The first, which is the more relevant for the discussion of cosmological instantons, is obtained by performing a Wick rotation of the time coordinate in the Minkowski-signature theory. We shall discuss this case first, and, afterwards, we shall consider an alternative Euclideansignature theory which is obtained by dimensionally reducing the original $D=11$ theory on $\mathrm{AdS}_{7}$ rather than $S^{7}$.

\subsection{The Wick-rotated Euclidean-signature theory}

The question of how Euclideanisation should be performed in the full supergravity theory is a thorny one, and in the literature one finds debate on the proper way to Euclideanise antisymmetric tensor fields, supersymmetry, etc. In particular, the Euclideanisation of the four-form giving rise to the cosmological constant has been a subject of much controversy [16, 17, 18, 19, 国. One opinion is that the electric components of an antisymmetric tensor field should be imaginary in the Euclidean regime, whereas the magnetic components should be real [37]. If one wants still to be able to consider electric/magnetic duality symmetries in the Euclidean regime, then having imaginary electric charges seems to be unavoidable [37]. For example, as observed in [38, 37, although real magnetically-charged extremal black holes can exist in a Euclideanised theory, electrically-charged extremal black holes can exist only if the charge, and hence the electric field, is imaginary. An alternative viewpoint [38, 18], in the original spirit of Belavin, Polyakov, Schwartz and Tyupkin [39] and 't Hooft 40] in their treatment of gauge field instantons, is that all components should be real so as to ensure that (with the possible exception of gravity itself) the Euclidean action will be

\footnotetext{
${ }^{3}$ One may well ask at this stage what significance should be attached to imaginary configurations of fields originally defined to be real. Since, in the quantum theory, fields are described by Hermitean operators whose eigenvalues are real, these complex configurations should not be thought of as expectation values of Hermitian operators but rather as off-diagonal matrix elements. For example, in Minkowski signature a self-dual (anti-self-dual) Maxwell field is complex and describes the wave function of a positive (negative) helicity photon i.e. the matrix element of the field operator between the vacuum and a one-particle state of definite helicity 35, 36.
} 
positive semidefinite and bounded below by the topological charge. This ensures that the Euclidean functional integrals will be gaussian.

If we adopt the first of these stances, then in order to Wick rotate the four-dimensional Lagrangian (2.3) to the Euclidean regime, we simply assume that it retains the identical form, but where the metric tensor is now positive definite. (In other words, $t \rightarrow-\mathrm{i} \sigma$ is viewed as a general coordinate transformation, with $\sigma$ subsequently taken to be real.) The equations of motion will therefore continue to be written as in (2.4). However, there will be a difference when we dualise the non-propagating 4-form $F_{(4)}$. Instead of (2.5), we should now solve for $F_{(4)}$ by writing

$$
F_{\mu \nu \rho \sigma}=\mathrm{i} c e^{6 \alpha \varphi} \epsilon_{\mu \nu \rho \sigma}
$$

where $c$ is real and $\epsilon_{\mu \nu \rho \sigma}$ is the real Levi-Civita tensor in the Euclidean regime. The factor of $\mathrm{i}$ is necessary since $F_{(4)}$ in four dimensions will necessarily have one index in the (Wickrotated) time direction, and so it will necessarily be electric in character. Following the standard procedure of substituting (4.8) into the remaining equations of motion, we find that they can be derived from the identical Lagrangian (2.6), with potential given by (2.7), as we obtained previously in the Minkowskian regime (with the understanding, as always, that the metric tensors appearing in (2.6) are now the Euclideanised ones).

Another piece of "supporting evidence" for the appropriateness of having imaginary electric fields is that by adopting this prescription, we ensure that the processes of Wick rotation and dualisation commute: Had we instead insisted that $F_{(4)}$ be dualised according to (2.5) rather than (4.8), we would have found the sign of the $c^{2}$ term in the potential (2.7) to be reversed. By contrast, if we simply Wick rotate the already-dualised Lagrangian given by (2.6) and (2.7), no such sign reversal occurs. By requiring that $F_{(4)}$ be imaginary in the Euclidean regime, we ensure that the order in which the dualisation and the Wick rotation are performed is immaterial. One satisfactory consequence of this is that, regardless of the order of dualisation and Wick rotation, a solution such as the $\operatorname{AdS}_{4} \times S^{7}$ solution in the original Minkowski-signature theory maps over into a sensible Euclideanised version.

A further consequence of taking the electric components of the field strength $F_{(4)}$, or, equivalently, the $A_{0 i j}$ components of the gauge potential $A_{(3)}$, to be imaginary in the Euclidean regime can be seen by looking at the Chern-Simons term $F_{(4)} \wedge F_{(4)} \wedge A_{(3)}$ in the eleven-dimensional action. Since the tensor density $\epsilon^{\mu_{1} \cdots \mu_{11}}= \pm 1,0$ is real in both the Minkowskian and Euclidean regimes, it follows that the Wick rotation introduces a factor of $\mathrm{i}$ in the Euclideanised Chern-Simons term, and this becomes consistent with having a real action if the electric components of $F_{(4)}$, and the $A_{0 i j}$ components of $A_{(3)}$, are imagi- 
nary. It is interesting to note that the various arguments that can be presented in support of having imaginary electric fields in the Euclidean regime all seem to revolve around the related notions of extremality, supersymmetry and duality.

The metric ansatz for the instanton solution in a Euclidean-signatured space takes the form

$$
d s_{4}^{2}=d \sigma^{2}+b(\sigma)^{2} d \Omega_{3}^{2} .
$$

The equations of motion are then given by [四]

$$
\varphi^{\prime \prime}+3 \varphi^{\prime} \frac{b^{\prime}}{b}=V_{, \varphi}(\varphi), \quad b^{\prime \prime}=-\frac{1}{6} b\left(\varphi^{\prime 2}+V(\varphi)\right)
$$

together with the first-order constraint

$$
\left(\frac{b^{\prime}}{b}\right)^{2}-\frac{1}{b^{2}}=\frac{1}{12} \varphi^{\prime 2}-\frac{1}{6} V .
$$

As discussed above, the potential $V(\varphi)$ is given by (2.7) as in the case of a Minkowskian spacetime. Note that the equations of motion in the Euclidean-signatured space can be obtained from those in the Minkowskian-signatured spacetime by making the Wick rotation $\tau=-i \sigma$. For both cases, we follow [1] and require that at $\sigma=0$ the potential vanishes, and that in the vicinity of $\sigma=0$, we have

$$
\varphi(0) \geq \varphi_{0}, \quad \varphi^{\prime}(0)=0, \quad b=\sigma .
$$

It follows from (4.10) that we will have $\varphi^{\prime \prime}\left(0_{+}\right)=V_{, \varphi}\left(\varphi\left(0_{+}\right)\right)>0$. This implies that $\left(\varphi-\varphi_{0}\right)$ is positive, and increases as $\sigma$ increases. Consequently, $V(\phi)$ is always positive and increases with $\sigma$, implying that $b^{\prime \prime}$ is always negative. Thus the initial velocity $b^{\prime}(0)=1$ reduces with increasing $\sigma$, and inevitablyf becomes zero at the point where

$$
b^{\prime 2}=b^{2}\left(\frac{1}{12} \varphi^{\prime 2}+\frac{1}{b^{2}}-\frac{1}{6} V\right)=0
$$

Since the acceleration $b^{\prime \prime}$ is still negative, this is a turning point at which the velocity $b^{\prime}$ reverses sign and starts to increase, driving the metric to a singularity at which $b \rightarrow 0$ for some finite $\sigma_{f}$. This evolutionary scenario was discussed in [1], where analytic continuations were made in order to view the solution as describing an open inflationary universe.

\footnotetext{
${ }^{4}$ The inevitability is due to the exponential increase of the potential $V(\varphi)$ at large $\varphi$. If the scale size $b$ would expand forever, then it implies that at $\sigma \rightarrow \infty$, we would have $\varphi \sim \tilde{c} \log \sigma$ where $c$ is a positive constant. Then we find that the left-hand sides of the equations 4.10 vanish, while the right-hand sides diverge at large $\sigma$, for our value of $V(\varphi)$.
} 
In [1], an assumption was made that the contribution of the potential $V(\varphi)$ becomes negligible near the singularity, and thereby it was argued that the solution near the singularity is of the form

$$
b \sim\left(\sigma_{f}-\sigma\right)^{\frac{1}{3}}, \quad \varphi^{\prime} \sim \frac{2}{\sqrt{3}}\left(\sigma_{f}-\sigma\right)^{-1} .
$$

This solution is independent of the detailed structure of $V(\varphi)$, and assumes only that it is monotonically increasing, but negligible near the singularity. However, this is not true in our case, where the potential $V(\varphi)$ is given by (2.7), arising from the $S O(8)$-invariant $S^{7}$ reduction of eleven-dimensional supergravity, even though $V(\varphi)$ does increase monotonically in the regime $\left(\varphi-\varphi_{0}\right)>0$. To see this, we may substitute the putative solution (4.14) into $V(\varphi)$, to see how it behaves near the singularity. We find

$$
V(\varphi) \sim V_{, \varphi}(\varphi) \sim\left(\sigma_{f}-\sigma\right)^{-2 \sqrt{7 / 3}}
$$

This in fact diverges more rapidly than any of the other terms in the equations of motion (4.10) and (4.11), as $\sigma$ approaches $\sigma_{f}$. In other words, the HT assumption that the contribution from $V(\varphi)$ can be neglected near $\sigma=\sigma_{f}$ is invalid in this case. In fact, in order for the HT solution (4.14) to be valid, the potential $V(\varphi)$, which will be of order $e^{a \varphi}$ at large $\varphi$, should have $a^{2}<3$, and this is not the case for the potential in our Lagrangian.

To solve the equations with our potential $V(\varphi)$, we note that for large $\varphi$ we have $V(\varphi) \sim \frac{1}{2} c^{2} e^{6 \alpha \varphi}$. Assuming that the dilaton diverges logarithmically at $\sigma_{f}$, the solution is then given by

$$
b \sim\left(\sigma_{f}-\sigma\right)^{\frac{1}{7}}, \quad e^{-3 \alpha \varphi} \sim \frac{7}{4} c\left(\sigma_{f}-\sigma\right) .
$$

(For a generic potential of the form $V(\varphi) \sim \frac{1}{2} c^{2} e^{a \varphi}$ with $a \geq \sqrt{3}$ at large $\varphi$, we have $b \sim\left(r_{f}-r\right)^{1 /\left(a^{2}\right)}$ and $e^{-a \varphi} \sim a^{2} c /\left(2 \sqrt{a^{2}-3}\right)\left(\sigma_{f}-\sigma\right)$.) Thus we see that the solution (4.16), like the solution (4.14), has a singularity at $\sigma=\sigma_{f}$, but the degree of the singularity is different from the one given in [1].

Note that, owing to the fact that $V(\varphi)$ increases monotonically in the regime $\left(\varphi-\varphi_{0}\right)>$ 0 , the scalar will be driven to positive infinity with velocity $\varphi^{\prime} \geq 0$. This is because even if the damping effect resulting from a positive $b^{\prime}$ were to tend to reduce $\varphi^{\prime}$ to zero, it nevertheless would follow from (4.10) that the acceleration $\varphi^{\prime \prime}$ would again be positive at this point. Thus, the velocity $\varphi^{\prime}$ never becomes negative. In fact, the potential $V(\varphi)$ monotonically increases in the regime $\varphi>\varphi_{\text {AdS }}$. It follows that the structure of the end-point singularity is independent of the initial $\varphi(0)$, as long as it is greater than $\varphi_{\text {Ads }}$. However, the initial behaviour of the solution does depend on $\varphi(0)$. If we choose the initial $\varphi(0)$ such that $V(\varphi(0)) \geq 0$, then $b^{\prime \prime}$ is always negative. On the other hand, if we choose $\varphi(0)$ such that 
$V(\varphi(0))<0$, then the initially we have $b^{\prime \prime}>0$, but it will quickly become negative, and will eventually reverse the expansion of the universe so as to shrink into the singularity.

Having obtained the four-dimensional cosmological instanton solution, it is of interest to examine it from the eleven-dimensional point of view. Near the initial time $\sigma \sim 0$, the eleven-dimensional metric approaches $E^{4} \times S^{7}$. Near the end of universe, when $\sigma \sim \sigma_{f}$, the eleven-dimensional metric is given by

$$
\begin{aligned}
d s_{11}^{2} & =e^{2 \alpha \varphi}\left(d \sigma^{2}+b^{2} d \Omega_{3}^{2}\right)+e^{2 \beta \varphi} d s_{7}^{2} \\
& \sim\left(\sigma_{f}-\sigma\right)^{-2 / 3}\left(d \sigma^{2}+\left(\sigma_{f}-\sigma\right)^{\frac{2}{7}} d \Omega_{3}^{2}\right)+\left(\sigma_{f}-\sigma\right)^{\frac{2}{21}} d s_{7}^{2}
\end{aligned}
$$

Thus we see that, owing to the fact that the dilaton diverges towards $+\infty$ as the final singularity is approached, the size of the internal seven-sphere $S^{7}$ shrinks to zero, providing a spontaneous compactification. Note that although the size of the three-sphere $d \Omega_{3}^{2}$ shrinks also in the 4-dimensional Einstein metric that we discussed earlier, its size in the elevendimensional metric in fact tends to infinity. Defining a co-moving time $\rho \sim\left(\sigma_{f}-\sigma\right)^{2 / 3} \rightarrow 0$ in $D=11$, the eleven-dimensional metric can be written as

$$
d s_{11}^{2} \sim d \rho^{2}+\rho^{-\frac{4}{7}} d \Omega_{3}^{2}+\rho^{\frac{1}{7}} d s_{7}^{2},
$$

from which we can extract a 4-dimensional metric $d s_{\mathrm{M}}^{2}$ using $d s_{11}^{2}=d s_{\mathrm{M}}^{2}+e^{2 \beta \varphi} d s_{7}^{2}$ (i.e. $\left.d s_{\mathrm{M}}=e^{2 \alpha \varphi} d s_{\text {Ein }}^{2}\right)$, giving

$$
d s_{\mathrm{M}}^{2} \sim d \rho^{2}+\rho^{-\frac{4}{7}} d \Omega_{3}^{2},
$$

in terms of which the three-sphere expands as $\rho \rightarrow 0$.

We may also study the instanton solutions in the four-dimensional theory obtained by reducing $D=11$ supergravity on the $S U(4) \times U(1)$ invariant squashed 7 -sphere. In this case, the solution will be supported by all the scalars that are singlets under $S U(4) \times U(1)$. It is worth noting that the 3 -form field strength $F_{(3)}$ can be dualised in $D=4$ to give another scalar field. However, this would be of axionic type, and would come with a dilatonic prefactor, so it would seem not to fit the bill for the "inflaton" in the Hawking-Turok model. However, let us consider the equations of motion for an instanton solution. Take the Lagrangian (2.20), obtained by reducing from $D=11$ on the squashed $S^{7}$. Note that we use the already-rotated dilatons, but we omit the tildes. Also, we dualise the 4 -form $F_{(4)}$ to a cosmological term with "charge" $c$, and we dualise the 3 -form $F_{(3)}$ to give an axion $\chi$. Thus the Lagrangian in $D=4$ is

$$
e^{-1} \mathcal{L}_{4}=R-\frac{1}{2}(\partial \phi)^{2}-\frac{1}{2}(\partial \varphi)^{2}-\frac{1}{2} e^{\frac{4}{\sqrt{7}} \varphi+\frac{6}{\sqrt{21}} \phi}(\partial \chi)^{2}-\widetilde{V}(\phi, \varphi)
$$


where the scalar potential $\widetilde{V}(\phi, \varphi)$ is given by

$$
\widetilde{V}(\phi, \varphi)=\frac{1}{2} c^{2} e^{\sqrt{7} \varphi}+6 m^{2} e^{\frac{3}{\sqrt{7}} \varphi+\frac{8}{\sqrt{21}} \phi}-e^{\frac{3}{\sqrt{7}} \varphi+\frac{1}{\sqrt{21}} \phi} R_{6}
$$

The potential has a minimum given by

$$
\begin{aligned}
\tilde{V}_{\min } & =\tilde{V}\left(\varphi_{\text {AdS }}, \phi_{\text {AdS }}\right)=-\frac{4}{3} c^{2} e^{\frac{4}{\sqrt{7}} \varphi_{\text {AdS }}} \\
e^{\frac{7}{\sqrt{21}} \phi_{\text {AdS }}} & =\frac{R_{6}}{48 m^{2}}, \quad e^{\frac{4}{\sqrt{7}} \varphi_{\text {AdS }}}=\frac{36 m^{2}}{c^{2}}\left(\frac{R_{6}}{48 m^{2}}\right)^{\frac{8}{7}} .
\end{aligned}
$$

From this we can obtain the equations of motion for the instanton solution with the metric ansatz (4.1); they are given by

$$
\begin{aligned}
& 3 \frac{\ddot{b}}{b}=-\frac{1}{2} \dot{\phi}^{2}-\frac{1}{2} \dot{\varphi}^{2}-\frac{1}{2} e^{\frac{4}{\sqrt{7}} \varphi+\frac{6}{\sqrt{21}} \phi} \dot{\chi}^{2}+\frac{1}{2} \widetilde{V}(\phi, \varphi), \\
& \frac{\ddot{b}}{b}+2\left(\frac{\dot{b}}{b}\right)^{2}=-\frac{2}{b^{2}}+\frac{1}{2} \widetilde{V}(\phi, \varphi) \text {, } \\
& \ddot{\phi}+3 \frac{\dot{b}}{b} \dot{\phi}=\frac{3}{\sqrt{21}} e^{\frac{4}{\sqrt{7}} \varphi+\frac{6}{\sqrt{21}} \phi} \dot{\chi}^{2}-\widetilde{V}_{, \phi}(\phi, \varphi), \\
& \ddot{\varphi}+3 \frac{\dot{b}}{b} \dot{\varphi}=\frac{2}{\sqrt{7}} e^{\frac{4}{\sqrt{7}} \varphi+\frac{6}{\sqrt{21}} \phi} \dot{\chi}^{2}-\widetilde{V}_{, \varphi}(\phi, \varphi), \\
& \ddot{\chi}+3 \frac{\dot{b}}{b} \dot{\chi}=-\frac{6}{\sqrt{21}} \dot{\phi} \dot{\chi}-\frac{4}{\sqrt{7}} \dot{\varphi} \dot{\chi} \text {. }
\end{aligned}
$$

Note that we can solve the last equation straightforwardly:

$$
\dot{\chi}=k b^{-3} e^{-\frac{4}{\sqrt{7}} \varphi-\frac{6}{\sqrt{21}} \phi} .
$$

Substituting $\dot{\chi}$ into the above equations, we obtain

$$
\begin{aligned}
\ddot{b} & =-\frac{1}{6}\left(\dot{\phi}^{2}+\dot{\varphi}^{2}+k^{2} b^{-6} e^{-\frac{4}{\sqrt{7}} \varphi-\frac{6}{\sqrt{21}} \phi}-\frac{1}{2} \widetilde{V}(\phi, \varphi)\right), \\
\ddot{\phi}+3 \frac{\dot{b}}{\dot{b}} \dot{\phi} & =\frac{3 k^{2}}{\sqrt{21}} b^{-6} e^{-\frac{4}{\sqrt{7}} \varphi-\frac{6}{\sqrt{21}} \phi}-\widetilde{V}_{, \phi}(\phi, \varphi), \\
\ddot{\varphi}+3 \frac{\dot{b}}{b} \dot{\varphi} & =\frac{2 k^{2}}{\sqrt{7}} b^{-6} e^{-\frac{4}{\sqrt{7}} \varphi-\frac{6}{\sqrt{21}} \phi}-\widetilde{V}_{, \varphi}(\phi, \varphi),
\end{aligned}
$$

together with the first-order constraint

$$
\left(\frac{\dot{b}}{b}\right)^{2}+\frac{1}{b^{2}}=\frac{1}{12} \dot{\phi}^{2}+\frac{1}{12} \dot{\varphi}^{2}+\frac{1}{12} k^{2} b^{-6} e^{-\frac{4}{\sqrt{7}} \varphi-\frac{6}{\sqrt{21}} \phi}+\frac{1}{6} \widetilde{V}(\phi, \varphi) .
$$

The contribution of the axion $\chi$ seems to force the solution to be singular at initial $\tau=0$. Here we again assume at $\tau=0$, we have $(\varphi, \phi)=\left(\varphi_{0}, \phi_{0}\right)$ and $(\dot{\varphi}, \dot{\phi})=(0,0)$, and $\widetilde{V}\left(\varphi_{0}, \phi_{0}\right)=0$. Assuming that the universe scale size $b$ is initially infinitesimal, then at small $\tau$ we will have $b \sim \tau^{1 / 3}$, which implies a curvature singularity of the order $\tau^{-2}$. If we 
set $k=0$, then the initial universe will be purely Euclidean, and the subsequent evolution will be determined by the two-scalar potential $\widetilde{V}(\varphi, \phi)$.

As a final note in this subsection, we may consider solutions where the unit three-sphere metric $d \Omega_{3}^{2}$ in the ansatz $(4.9)$ is replaced by a flat metric $d \vec{x}^{2}$. In this case, the equations can be solved exactly, and the solution turns out to be just the Euclideanisation of the domain wall discussed in section 3.1. This solution is now given (after a coordinate change on $y$ ) by

$$
\begin{aligned}
d s^{2} & =e^{2 B} d y^{2}+e^{2 A} d \vec{x}^{2}, \quad e^{-\frac{5}{\sqrt{7}} \varphi}=e^{-\frac{5}{\sqrt{7}} \varphi_{\text {AdS }}}+k|y| \\
e^{3 A} & =e^{-B}=\frac{5}{2 k}\left(\sqrt{\frac{6 R_{7}}{7}} H^{\frac{7}{10}}-c H^{\frac{3}{10}}\right) .
\end{aligned}
$$

If $k>0$ then $y$ runs from 0 , at which the solution is the hyperbolic space $H_{4}$, to infinity, at which the solution is flat. If $k<0$ then $y$ runs from 0 , at which the solution is $H_{4}$, to $y=-e^{-\frac{5}{\sqrt{7}} \varphi_{\mathrm{AdS}}} / k$, at which one finds a genuine curvature singularity. If $k=0$, then the solution is the hyperbolic space $H_{4}$ everywhere. As in our previous discussions of domain wall in Minkowski-signatured spaces, we can also consider solutions here where the value of $\varphi$ at $y=0$ differs from the one chosen in (4.27). Under these circumstances, the solution either reaches the $H_{4}$ form for some value of $|y|$ that is greater than zero, or else it fails to become $H_{4}$ by the time $y$ reaches zero. In either of these cases there is a delta-function curvature singularity at $y=0$, which can be thought of as a domain wall.

\subsection{Patching cosmological solutions with domain walls}

We have seen in the last subsection that one may find instanton solutions to Wick-rotated supergravity theories that share some of the features of the HT instanton. One salient feature of such instantons is the presence of a singularity. In order to avoid such singularities, a proposal was made in 41] to patch together non-singular regions of cosmological instanton solutions using a domain wall. We shall show that such patching can indeed be carried out consistently within the specific context of spherically-reduced $D=11$ supergravity as considered above. The needed domain wall for this construction has the same source action as that for the supersymmetric domain-wall solution presented in section 3.1, except that now we shall need to couple it to a (non-supersymmetric) instanton background (4.9), analytically continued back to Minkowski signature.

In the cosmological analyses of Refs [1, 41], the overall cosmological solution starts with an Euclidean instanton, which is followed until a surface of vanishing extrinsic curvature is reached, at which an analytic continuation back to a Minkowski-signature solution is made. 
To do this in our case, one may expand the instanton metric (4.9), writing

$$
d s_{4}^{2}=d \sigma^{2}+b(\sigma)^{2}\left(d \psi^{2}+\sin ^{2} \psi d \Omega_{2}^{2}\right)
$$

The surface of vanishing extrinsic curvature occurs at $\psi=\pi / 2$, at which one may continue back to a Minkowski-signature metric by letting $\psi=\pi / 2+\mathrm{i}$. After this analytic continuation, one has the Minkowski-signature metric

$$
d s_{4}^{2}=-b(\sigma)^{2} d t^{2}+d \sigma^{2}+b(\sigma)^{2} \cosh ^{2} t d \Omega_{2}^{2}
$$

which describes a universe with de Sitter-like features. The metric (4.29) describes only part of this spacetime, however, and needs a further analytic continuation in order to cover the whole spacetime. Analytic continuation in this way does not provide a refuge from the singularity, however. The singularity that we have seen in the last subsection at $\sigma \sim \sigma_{f}$ persists also in the analytically continued solution. Accordingly, in 41] a suggestion was made to simply cut out the singular portion of the spacetime by patching two non-singular regions of the form (4.29) together, with a domain wall located at the patching surface.

From the viewpoint of $D=11$ supergravity, the coupling of the needed domain wall to the background supergravity fields is described by including the action for a fundamental supermembrane [42] into the overall (field + source) action. Since we actually wish to make this coupling in the spherically-reduced $D=4$ theory discussed in section 2, we shall choose to let the membrane lie entirely in the non-compact $D=4$ spacetime, and shall also have to use the appropriate form of the background metric, as obtained from the spherical dimensional reduction. The bosonic part of the supermembrane action for a supermembrane located on the worldvolume hypersurface $X^{M}(\xi)(M=0,1, \ldots, 10)$ in a background described by the $D=11$ supergravity fields $g_{M N}, A_{M N P}$ is

$$
I_{\mathrm{memb}}^{(11)}=T \int d^{3} \xi\left\{\left[-\operatorname{det}\left(\partial_{i} X^{M} \partial_{j} X^{N} g_{M N}(X)\right)\right]^{1 / 2}+\frac{1}{6} \epsilon^{i j k} \partial_{i} X^{M} \partial_{j} X^{N} \partial_{k} X^{P} A_{M N P}\right\}
$$

In order to obtain the corresponding source action in $D=4$ supergravity, we shall adopt the spherical reduction ansatz (2.1) and shall restrict the membrane to lie in the $D=4$ subspace. To do this, we divide the $D=11$ coordinates $X^{M}$ into two ranges: $X^{M}=$ $\left(X^{\mu}, Y^{m} ; \mu=0,1,2,3 ; m=4, \ldots, 10\right)$ and then restrict the membrane coordinates by setting $Y^{m}=$ const. The supermembrane action then reduces to

$$
I_{\mathrm{memb}}^{(4)}=T \int d^{3} \xi\left\{e^{3 \alpha \varphi}\left[-\operatorname{det}\left(\partial_{i} X^{\mu} \partial_{j} X^{\nu} g_{\mu \nu}^{(4)}(X)\right)\right]^{1 / 2}+\frac{1}{6} \epsilon^{i j k} \partial_{i} X^{\mu} \partial_{j} X^{\nu} \partial_{k} X^{\rho} A_{\mu \nu \rho}\right\}
$$


where $g_{\mu \nu}^{(4)}$ is the $D=4$ metric defined in the reduction ansatz 2.1) and $\varphi$ is the breathingmode scalar field, also defined in (2.1).

The consistency of putting a membrane into a given background is determined by checking whether the "brane-wave" equations following from (4.31) upon varying the membrane coordinates $X^{M}$ are satisfied, plus verifying that charge conservation requirements are satisfied. The brane-wave equations derived from (4.31) are

$$
\begin{aligned}
& \partial_{i}\left(e^{3 \alpha \varphi} \sqrt{-\tilde{g}} \tilde{g}^{i j} \partial_{j} X^{\nu} g_{\rho \nu}^{(4)}(X)\right)-\frac{1}{2} e^{3 \alpha \varphi} \sqrt{-\tilde{g}} \tilde{g}^{i j} \partial_{i} X^{\mu} \partial_{j} X^{\nu} \partial_{\rho} g_{\mu \nu}^{(4)}(X) \\
& -3 \alpha \partial_{\rho} \varphi(X) e^{3 \alpha \varphi} \sqrt{-\tilde{g}}-\frac{1}{3 !} \epsilon^{i j k} \partial_{i} X^{\mu} \partial_{j} X^{\nu} \partial_{k} X^{\sigma} F_{\sigma \mu \nu \rho}(X) \\
& \quad=0
\end{aligned}
$$

where $\tilde{g}_{i j}=\partial_{i} X^{\mu} \partial_{j} X^{\nu} g_{\mu \nu}^{(4)}(X)$.

In order to solve the brane-wave equations in the background of the analytically continued instanton metric (4.29) (with the 4-form field strength now given again by (2.5), since we have analytically continued back to a Minkowski-signature region), we first pick an appropriate "static gauge" for the membrane worldvolume coordinates:

$$
\begin{aligned}
X^{t} & =\xi^{0} \\
X^{\theta} & =\xi^{1} \\
X^{\phi} & =\xi^{2}
\end{aligned}
$$

where $\theta$ and $\phi$ here are the angular coordinates for the $S^{2}$ part of the metric (4.29).

We now aim, following [41, to patch together two background solutions of the form (4.29) at an appropriate value of $\sigma$, which we shall call $\sigma_{m}$. Accordingly, we shall try to satisfy the brane-wave equations (4.32), together with the gauge conditions (4.33), by making an additional ansatz corresponding to a static spherical membrane at constant $\sigma$ :

$$
X^{\sigma}=\sigma_{m}
$$

The only non-trivial check is that of the brane-wave equation for $\rho=\sigma$ in (4.32), as the other three equations turn out to be automatically satisfied by virtue of gauge identities conjugate to the gauge conditions (4.33). Evaluating this one non-trivial equation, we find the matching condition

$$
\frac{3 b^{\prime}\left(\sigma_{m}\right)}{b\left(\sigma_{m}\right)}=-3 \alpha \varphi^{\prime}\left(\sigma_{m}\right) e^{3 \alpha \varphi\left(\sigma_{m}\right)}+c e^{6 \alpha \varphi\left(\sigma_{m}\right)},
$$

which imposes a relation between the membrane location $\sigma_{m}$ (4.34) and the background's integration constant $c$ appearing in (2.5). Note that since the analytically-continued instanton background (4.29) is not supersymmetric, one should not have expected a no-force 
condition for the included membrane (4.31), so it should come as no surprise that there is a specific consistent value $\sigma_{m}$ for our static membrane ansatz (4.34), dependent on the parameter $c$ determining the background.

Finally, we come to the charge-conservation requirement necessary for a consistent coupling of the membrane to the background (4.29). The presence of the source action modifies the antisymmetric-tensor field equation (2.4c) by the inclusion of a delta-function source term:

$$
d\left(e^{-6 \alpha \varphi} * F_{(4)}\right)=T * J_{(3)},
$$

where the delta-function source current is

$$
* J_{(3)}=\delta\left(\sigma-\sigma_{m}\right) d \sigma
$$

Clearly, we cannot satisfy this sourced field equation everywhere by the single condition (2.5) for a given integration constant $c$, because this single condition would cause the lefthand side of (4.36) to vanish. What we must instead do to achieve a consistent patching is to have different values of the integration constant $c$ on the two sides of the domain wall:

$$
e^{-6 \alpha \varphi} * F_{(4)}=-\left[c_{+} \theta\left(\sigma-\sigma_{m}\right)+c_{-} \theta\left(\sigma_{m}-\sigma\right)\right] .
$$

The charge conservation condition is now transparent: imposing the sourced antisymmetrictensor field equation (4.36) now yields the condition

$$
c_{-}-c_{+}=T,
$$

where $T$ is the membrane tension appearing in the source action (4.30, 4.31). Conditions 4.35, 4.39) thus define the $\sigma_{m}$ location and charge conservation requirements for a domain wall to be consistently inserted into an analytically-continued instanton background of the form (4.29). One should note that at the quantum level, the tension $T$ of the included membrane will need to be quantised in accordance with Dirac charge-quantisation conditions taken together with the web of duality relations for supergravity $p$-branes [43, 44, 45, 46]. These conditions require the membrane tension $T$ to sit on a discretised lattice of allowed values. Thus, the integration constants $c_{ \pm}$entering into the charge matching condition (4.39) will have to take values that respect the lattice of allowed $T$ values.

\subsection{The Euclidean-signature theory from $\mathrm{AdS}_{7}$ reduction}

Another way of obtaining a Euclidean-signature theory is to perform a dimensional reduction on a space that includes the time direction, instead of making a Wick rotation on the 
time direction. Although such Euclidean-signature solutions may not be directly relevant for vacuum tunnelling considerations such as those discussed in the previous two subsections, we shall nonetheless take the opportunity to discuss them here. Previous discussions of such reductions have principally focussed on reductions on Minkowski-signature tori. Such compactifications have been considered in connection with the classification of static p-brane solutions in terms of instanton solutions of non-compact sigma models 447, 48. Here, we shall instead be concerned with reduction on an AdS spacetime, as discussed in appendix A. Principally, we shall consider the reduction of $D=11$ supergravity on $\mathrm{AdS}_{7}$. The generalisation to the other cases, for example $D=11$ on $\mathrm{AdS}_{4}$ and type IIB on $\mathrm{AdS}_{5}$, is straightforward.

It is worth emphasising that the Euclidean-signature supergravities obtained by making timelike reductions from standard Minkowskian-signature supergravities are themselves perfectly real, and, by contrast with the Euclidean-signature theories obtained by Wick rotation, all fields should be taken to be real. In particular, the notions of extremality, duality and supersymmetry will now all require that the fields of the Euclidean-signature theory be real (see, for example, 48]).

From the results in appendix $\mathrm{A}$, we see that after dualising the 4-form field strength the four-dimensional Lagrangian will be given by

$$
e^{-1} \mathcal{L}=R-\frac{1}{2}(\partial \varphi)^{2}-\tilde{V}(\varphi)
$$

where the potential $\widetilde{V}(\varphi)$ is given by

$$
\widetilde{V}(\varphi)=-\frac{1}{2} c^{2} e^{6 \alpha \varphi}-e^{\frac{18}{7} \alpha \varphi} R_{7}
$$

Comparing with the potential $V(\varphi)$ for the usual theory obtained by dimensional reduction on $S^{7}$, given in (2.7), we see that the sign of the $c^{2}$ term here is reversed. In addition, although the $R_{7}$ term is identical in form, it should be recalled that $R_{7}$ is negative in (4.41), since it is now the Ricci scalar of the $\mathrm{AdS}_{7}$ space on which the dimensional reduction is being performed. In fact, the net effect is therefore simply to reverse the sign of the scalar potential that we considered previously. Thus $\tilde{V}$ now has a maximum $\widetilde{V}_{\max }=-V_{\min }>0$. This is true generally: the scalar potential for the breathing mode in supergravity compactified on an AdS is opposite in sign to that arising in the compactification on a sphere.

In this case, we have $\varphi^{\prime \prime}\left(0_{+}\right)=\widetilde{V}_{, \varphi}\left(\varphi_{0}\right)<0$. Thus $\left(\varphi-\varphi_{0}\right)$ in this case becomes negative as $\sigma$ increases. The potential $\widetilde{V}(\varphi)$ is again always positive, implying that the acceleration $b^{\prime \prime}$ is always negative. Thus the velocity $b^{\prime}$ will decrease. Whether the end of the universe 
is singular or not depends on whether the dilaton stabilises. We note that the first-order constraint (4.11) implies in this case that

$$
\left(\frac{b^{\prime}}{b}\right)^{2}=\frac{1}{12} \varphi^{\prime 2}+\frac{1}{b^{2}}-\frac{1}{6} \widetilde{V}(\varphi)
$$

Since in the region $\left(\varphi-\varphi_{0}\right)<0$, the potential $\tilde{V}(\varphi)$ is positive, it is possible that the velocity $b^{\prime}$ can reach zero at some finite time, and the negative acceleration $b^{\prime \prime}$ then implies that the universe will eventually shrink down to zero size at finite $\sigma_{f}$. When this happens, there are two possible outcomes. The first one is that the dilaton diverges, and runs to $-\infty$, in which case the potential vanishes. The solution near $\sigma_{f}$ is then given by

$$
b \sim\left(\sigma_{f}-\sigma\right)^{\frac{1}{3}}, \quad \varphi^{\prime} \sim-\frac{2}{\sqrt{3}}\left(\sigma_{f}-\sigma\right)^{-1}
$$

and hence the universe ends up in a singularity.

However, there can be a non-singular end-point as well. Note that $\widetilde{V}(\varphi)$ does not increase monotonically for $\left(\varphi-\varphi_{0}\right)<0$; in fact it decreases when $\varphi$ passes the minimum point $\varphi_{\text {AdS }}<\varphi_{0}$. This implies that $\varphi^{\prime \prime}$ can be positive, and hence can cause the value of $\varphi$ to increase. The net effect is that $\varphi$ eventually stabilises at $\varphi_{\text {Ads }}$. In this case we eventually have $b^{\prime \prime}=-\frac{1}{6} \widetilde{V}_{\max } b$, and so

$$
b \sim \sin \left(\sqrt{\frac{1}{6} \widetilde{V}_{\max }}\left(\sigma_{f}-\sigma\right)\right) .
$$

In this case, the "end of the universe" is an $S^{4}$. The solution has no singularity at $\sigma_{f}$; in fact the metric becomes purely Euclidean. The universe afterwards becomes periodic.

A priori, one would expect that if $\widetilde{V}_{\max }$ were sufficiently small, such that $b^{\prime}$ never reached zero, then the universe would expand forever. In this case $\left(\varphi-\varphi_{0}\right)$ would be driven to negative infinity, since we have seen above that the stabilisation of $\varphi$ implies that $b^{\prime}$ becomes negative after a certain amount of time. Whether this possibility arises or not depends on whether there is such a solution. It is easy to verify that the solution exists only for potentials $\widetilde{V} \sim \frac{1}{2} g^{2} e^{a \varphi}$ with $a^{2}<1 / 2$. To see this, we note that (4.10) leads to a solution that asymptotically approaches

$$
b \sim \tau^{\frac{1}{a^{2}}}, \quad e^{a \phi} \sim \frac{2 \sqrt{3-a^{2}}}{a^{2} g} \tau^{-1},
$$

where $\tau=i \sigma$. The first-order constraint (4.11) implies that only if $a^{2}<1 / 2$ can the $1 / b^{2}$ contribution can be ignored. In our case we have $\tilde{V}(\varphi) \sim R_{7} e^{18 \alpha \varphi / 7}=R_{7} e^{3 \varphi / \sqrt{7}}$, and hence such a solution does not occur here.

Thus we see that there are two possible evolutionary scenarios in this AdS reduction case, depending presumably on the parameters $c$ and $R_{7}$ in the potential. If the slope $\widetilde{V}_{, \varphi}$ 
is sufficiently mild, then $\varphi$ will stabilise at $\varphi_{\text {AdS }}$, and the universe will become periodic, without a singularity. On the other hand if the slope is steeper then $\varphi$ will be driven to negative infinity, and consequently the universe will end up at the singular point $\sigma_{f}$, with a near-singularity solution given by (4.14).

So far, we have studied the solution in terms of the four-dimensional Einstein metric $d s_{\operatorname{Ein}}^{2}$. In terms of the eleven-dimensional metric, the first possibility above, where the dilaton stabilises to $\varphi=\varphi_{\text {AdS }}$ and $b$ is given by (4.44), the size of the internal $\mathrm{AdS}_{7}$ space stabilises also. The eleven-dimensional metric approaches the form

$$
d s_{11}^{2} \sim d \sigma^{2}+\sin ^{2}\left(\sqrt{\frac{1}{6} \widetilde{V}_{\min }}\left(\sigma_{f}-\sigma\right)\right) d \Omega_{3}^{2}+d s_{7}^{2}
$$

when $\sigma \rightarrow \sigma_{f}$. In the second possibility, given by (4.45), the dilaton is instead driven to negative infinity, and hence the "internal" AdS space becomes infinite in size. The elevendimensional metric is given by

$$
\begin{aligned}
d s_{11}^{2} & \sim\left(\sigma_{f}-\sigma\right)^{\frac{2 \sqrt{7}}{3 \sqrt{3}}} d \sigma^{2}+\left(\sigma_{f}-\sigma\right)^{\frac{2}{3}(1+\sqrt{7 / 3})} d \Omega_{3}^{2}+\left(\sigma_{f}-\sigma\right)^{-\frac{4}{3 \sqrt{21}}} d s_{7}^{2} \\
& \sim d \rho^{2}+\rho^{\frac{2(\sqrt{3}+\sqrt{7})}{3 \sqrt{3}+\sqrt{7}}} d \Omega_{3}^{2}+\rho^{-\frac{4}{7+3 \sqrt{21}}} d s_{7}^{2}
\end{aligned}
$$

where $\rho \sim\left(\sigma_{f}-\sigma\right)^{1+\sqrt{7 / 27}}$. Thus from the eleven-dimensional point of view, this solution really describes an expanding eight-dimensional universe.

\section{Conclusions}

In this paper, we have considered various Kaluza-Klein dimensional reductions of $D=11$ supergravity and type IIB supergravity, focussing principally on the cases where the internal space is a sphere. The goal was to construct lower-dimensional Lagrangians that could be used for describing cosmological instanton and domain-wall solutions. In order that these should also be solutions of the original $D=11$ or type IIB supergravities, it is important that the dimensional reduction procedure be a consistent one. We achieved this by making a truncation to the subset of Kaluza-Klein modes that are the singlets under a transitivelyacting symmetry group of the compactifying sphere. Our main examples were the $S^{7}$ and $S^{4}$ reductions of $D=11$ supergravity, and the $S^{5}$ reduction of type IIB supergravity, since in all these cases there exist anti-de Sitter "vacuum" solutions in the lower-dimensional spacetime. The simplest consistent truncations arise when one just retains the singlets under the $S O(n+1)$ isometry group of the $n$-sphere. In the gravitational sector this corresponds to keeping only the metric and the breathing-mode scalar in the lower-dimensional theory. In 
the case of spheres of odd dimension, a slightly enlarged consistent truncation is possible, where one keeps the singlets under the $S U(m+1) \times U(1)$ subgroup of the $S O(2 m+2)$ isometry group of the "round" $(2 m+1)$-sphere. In the gravitational sector this has the effect of including a further scalar mode in the lower-dimensional theory, which parameterises the homogeneous "squashing" along the Hopf fibres of $S^{2 m+1}$, viewed as a $U(1)$ bundle over $C P^{m}$. We also considered other consistent reductions, on $S^{2}, S^{3}$ and AdS spaces.

Having obtained the consistently-truncated lower dimensional theories, we then studied solutions of two kinds. One class consisted of supersymmetric domain-wall solutions, also known as $(D-2)$-branes. An interesting feature of these solutions is that, once oxidised back to the original higher-dimensional theory, they become standard isotropic $p$-branes. In fact the domain wall can be understood as arising from performing a dimensional reduction on the spheres that foliate the space transverse to the isotropic $p$-brane. For example, we found a domain wall (i.e. a membrane) in $D=4$, which can be interpreted as coming from the dimensional reduction of the eleven-dimensional M2-brane on the 7-spheres that foliate its transverse space.

The other class of solutions that we considered consisted of instanton solutions of the Euclideanised theories, that could, in the spirit of [1, 4], be interpreted via appropriate analytic continuations as describing open universes. Our analysis of the instanton solutions concentrated on the four-dimensional case of greatest phenomenological interest. In our solutions the rôle of the inflaton is played by the breathing-mode scalar of the spherical reduction, and hence the form of the scalar potential is precisely determined. This potential is similar to the sorts of potential that were postulated in 迎, 侚, and in particular one finds singular instanton solutions of the $S O(4)$-invariant deformed $S^{4}$ kind. However, the details of the solutions, and in particular the nature of the singularity, are somewhat different from the generic ones obtained in [1, 勾, owing to the fact that certain of their assumptions about the asymptotic form of the scalar potential are not satisfied by the explicit potential that we obtained. This is reflected in the fact that the scale factor $b(\sigma)$ in the instanton metric (4.9) near the singularity is of the form $b \sim\left(\sigma_{f}-\sigma\right)^{1 / 7}$ rather than of the form $b \sim\left(\sigma_{f}-\sigma\right)^{1 / 3}$ which was found in $[1, \llbracket$. The precise origin of the inflaton mode in the 7-sphere compactification of $D=11$ supergravity discussed in [4] is not specified. It is presumably to be thought of as one of the scalars coming from the Kaluza-Klein reduction, although from its coupling to the 4-form it is evidently not the breathing mode. It is interesting that the outcome from our analysis where we do use the breathing mode as the inflaton, allowing us to give an explicit form for its scalar potential, is a singular instanton 
solution exhibiting many of the same features that were obtained in [1, 过 for generic forms of a postulated potential. The specific potentials that we have obtained in this paper give only a very small amount of inflation, however. Thus, the search for realistic inflationary models based on a fundamental underlying theory remains an open subject. 


\section{Acknowledgements and Note Added}

We are grateful for discussions with Raphael Bousso, Gary Gibbons, Stephen Hawking, André Linde and Neil Turok. We would specifically like to acknowledge discussions with Rapael Bousso, Stephen Hawking and André Linde that led to clarification of the amount of inflation that can be expected in the type of model considered in this paper. After distributing this paper, we received Ref. [52], which also addresses some of the issues discussed in the present work.

\section{Appendices}

\section{A General sphere and AdS reductions}

Let us consider the general ansatz for a "breathing-mode" reduction on an internal space $\mathcal{Y}$ which is allowed to be either a sphere, or an anti-de Sitter spacetime, or, indeed, any other Einstein space or spacetime, with positive, negative or zero Ricci scalar. In the case of reductions on AdS or other spacetimes, the time coordinate itself forms part of the internal space, and the resulting lower-dimensional theory is of Euclidean signature. We shall begin by considering the reduction of a pure Einstein theory in $D=d_{x}+d_{y}$ dimensions, where the lower-dimensional theory has coordinates $x^{\mu}$ and dimension $d_{x}$, while the internal space $\mathcal{Y}$ has coordinates $y^{m}$ and dimension $d_{y}$. The reduction ansatz for the $D$-dimensional metric is

$$
d \hat{s}^{2}=e^{2 \alpha \varphi} d s_{x}^{2}+e^{2 \beta \varphi} d s_{y}^{2},
$$

where the subscripts $x$ and $y$ indicate the lower-dimensional space and the compactifying space respectively. We find that the reduced action will have a canonical pure EinsteinHilbert form for the metric if the constants $\alpha$ and $\beta$ satisfy $\alpha\left(d_{x}-2\right)+\beta d_{y}=0$. The normalisation of the kinetic term for $\varphi$ will then be canonical if we choose $\alpha$ and $\beta$ to be given by

$$
\alpha^{2}=\frac{d_{y}}{2\left(d_{x}-2\right)\left(d_{x}+d_{y}-2\right)}, \quad \beta=-\frac{\alpha\left(d_{x}-2\right)}{d_{y}} .
$$

Then we have the following result for the reduction of the higher-dimensional EinsteinHilbert Lagrangian:

$$
\hat{e} \hat{R}=\sqrt{g_{y}} \mathcal{L}_{x}
$$

where $g_{y}$ denotes the metric on the $d_{y}$-dimensional space, and

$$
e^{-1} \mathcal{L}_{x}=R-\frac{1}{2}(\partial \varphi)^{2}+e^{2(\alpha-\beta) \varphi} R_{y}-\alpha\left(d_{x}-3\right) \square \varphi .
$$


The last term in (A.4) is just a total divergence, and so it can be dropped.

For future reference, we record also the expressions for the vielbein components of the Ricci tensor of the metric $d \hat{s}^{2}$, given in terms of the lower-dimensional quantities for the $x$-space and $y$-space metrics in (A.1). After using the expressions for $\alpha$ and $\beta$ given in (A.2), we find that the vielbein components of the Ricci tensor are

$$
\begin{aligned}
\hat{R}_{\mu \nu} & =e^{-2 \alpha \varphi}\left(R_{\mu \nu}-\alpha \square \varphi \eta_{\mu \nu}-\frac{1}{2} \partial_{\mu} \varphi \partial_{\nu} \varphi\right), \\
\hat{R}_{m n} & =e^{-2 \beta \varphi} R_{m n}-\beta e^{-2 \alpha \varphi} \square \varphi \eta_{m n}, \\
\hat{R}_{\mu n} & =0 .
\end{aligned}
$$

(The signatures for the local Lorentz metrics $\eta_{\mu \nu}$ and $\eta_{m n}$ are to be chosen appropriately, depending upon whether a spacelike or a timelike reduction is being considered.)

The above discussion may be augmented by including a field strength $\hat{F}_{n}$ of degree $n$ in the original $D$-dimensional theory, so that we now start from the Lagrangian

$$
\hat{\mathcal{L}}=\hat{e} \hat{R}-\frac{1}{2 n !} \hat{e} \hat{F}_{n}^{2} .
$$

There are two possible reductions, within the framework of the consistently-truncated theories that we are considering, where only singlets under the isometry group of the internal space are retained. In the generic case, where the degree $n$ is not equal to $d_{y}$, the metric reduction ansatz (A.1) will be supplemented by the ansatz

$$
\hat{F}_{n}(x, y)=F_{n}(x) .
$$

On the other hand, in the special case that $n=d_{y}$, the more general ansatz

$$
\hat{F}_{n}(x, y)=F_{n}(x)+m \epsilon_{d_{y}},
$$

can be made, where $m$ is a constant and $\epsilon_{d_{y}}$ is the volume form on the internal space. The dimensionally-reduced Lagrangian now takes the form

$$
e^{-1} \mathcal{L}_{x}=R-\frac{1}{2}(\partial \varphi)^{2}+e^{2(\alpha-\beta) \varphi} R_{y}-\frac{1}{2 n !} e^{-2(n-1) \alpha \varphi} F_{n}^{2}-\frac{1}{2} \zeta m^{2} e^{2\left(d_{x}-1\right) \alpha \varphi},
$$

where $\zeta=+1$ if the internal space $\mathcal{Y}$ has Euclidean signature, and $\zeta=-1$ if $\mathcal{Y}$ has Minkowski signature. The last term is present only if $d_{y}=n$ and the more general ansatz (A.9) is made.

\footnotetext{
${ }^{5}$ If the internal space $\mathcal{Y}$ is not a sphere or AdS, it may be that it has additional invariant tensors aside from the volume form. In such cases, a consistent singlet reduction can be performed in which $\epsilon_{d_{y}}$ in (A.9) is replaced by the invariant tensor. For instance, if $\mathcal{Y}$ is a Kähler manifold then the Kähler form, or powers of the Kähler form, can be used. Examples of this kind arise in the squashed $S^{7}$ and $S^{5}$ reductions considered in sections 2.2 and 2.5 .
} 
A case of particular interest arises when $d_{x}$ is equal to $n$. We can then dualise the field strength $F_{n}$, which will have no degrees of freedom, to give a cosmological-type term. Specifically, we will have

$$
F_{n}=m e^{2(n-1) \alpha \varphi} \epsilon_{d_{x}}
$$

The two cases $d_{x}=n$ or $d_{y}=n$ can then be treated in a symmetrical way, by taking $F_{n}=0$ in the latter case. The equations of motion in the lower dimension will then be

$$
\begin{aligned}
R_{\mu \nu} & =\frac{1}{2} \partial_{\mu} \varphi \partial_{\nu} \varphi-\frac{1}{d_{x}-2} R_{y} e^{2(\alpha-\beta) \varphi} g_{\mu \nu}+\frac{\zeta m^{2}}{2\left(d_{x}-2\right)} e^{2\left(d_{x}-1\right) \alpha \varphi} g_{\mu \nu}, \\
\square \varphi & =-2(\alpha-\beta) R_{y} e^{2(\alpha-\beta) \varphi}+\zeta m^{2}\left(d_{x}-1\right) \alpha e^{2\left(d_{x}-1\right) \alpha \varphi} .
\end{aligned}
$$

These can be derived from the Lagrangian

$$
e^{-1} \mathcal{L}_{x}=R-\frac{1}{2}(\partial \varphi)^{2}+e^{2(\alpha-\beta) \varphi} R_{y}-\frac{1}{2} \zeta m^{2} e^{2\left(d_{x}-1\right) \alpha \varphi} .
$$

\section{B A general class of domain walls}

First, consider a general class of Lagrangians in $D$ dimensions of the form

$$
e^{-1} \mathcal{L}=R-\frac{1}{2}(\partial \varphi)^{2}-V(\varphi)
$$

with the potential $V(\varphi)$ given by

$$
V(\varphi)=\frac{1}{2} g_{1}^{2} e^{a_{1} \varphi}-\frac{1}{2} g_{2}^{2} e^{a_{2} \varphi}-\lambda g_{1} g_{2} e^{\frac{1}{2}\left(a_{2}+a_{1}\right) \varphi} .
$$

If we look for domain-wall solutions where the metric is

$$
d s^{2}=e^{2 A} d x^{\mu} d x_{\mu}+e^{2 B} d y^{2}
$$

where $A$ and $B$ are functions only of $y$, the equations of motion are

$$
\begin{aligned}
& \varphi^{\prime \prime}+\left(d A^{\prime}-B^{\prime}\right) \varphi^{\prime}=V_{, \varphi}(\varphi) \\
& A^{\prime \prime}+\left(d A^{\prime}-B^{\prime}\right) A^{\prime}=d A^{\prime \prime}+d\left(A^{\prime}-B^{\prime}\right) A^{\prime}+\frac{1}{2} \varphi^{\prime 2}=-\frac{V(\varphi)}{D-2},
\end{aligned}
$$

where we have $d=D-1$. The equations can be solved by making the ansatz

$$
\begin{aligned}
& \varphi^{\prime}=b_{1} e^{\frac{1}{2} a_{1} \varphi+B}+b_{2} e^{\frac{1}{2} a_{2} \varphi+B}, \\
& A^{\prime}=c_{1} e^{\frac{1}{2} a_{1} \varphi+B}+c_{2} e^{\frac{1}{2} a_{2} \varphi+B},
\end{aligned}
$$

for constants $b_{1}, b_{2}, c_{1}, c_{2}$, provided that $\lambda$ satisfies

$$
\lambda^{2}=-\frac{\left((D-2) a_{1} a_{2}-2(D-1)\right)^{2}}{\left[(D-2) a_{1}^{2}-2(D-1)\right]\left[(D-2) a_{2}^{2}-2(D-1)\right]} .
$$


This fist-order ansatz for the solutions is inspired by the equations that come from requiring supersymmetry in those cases where (B.1) is part of a supergravity Lagrangian. We have encountered several examples in this paper where such first-order equations are indeed seen to arise in this way.

The dilaton coupling constants $a_{1}$ and $a_{2}$ that we consider in this paper satisfy

$$
a_{1} a_{2}=\frac{2(D-1)}{D-2}
$$

and therefore $\lambda=0$. The domain-wall solutions with non-vanishing $\lambda$ were constructed in [49] for gauged supergravities in $D=7$ [50] and $D=6$ [51]. Substituting the ansatz to the equations of motion, we find that the four constants are given by

$$
\begin{aligned}
b_{1}^{2}=\frac{(D-2) a_{1}^{2} g_{1}^{2}}{(D-2) a_{1}^{2}-2(D-1)}, & c_{1}=-\frac{b_{1}}{(D-2) a_{1}}, \\
b_{2}^{2}=-\frac{(D-2) a_{2}^{2} g_{2}^{2}}{(D-2) a_{2}^{2}-2(D-1)}, & c_{2}=-\frac{b_{2}}{(D-2) a_{2}} .
\end{aligned}
$$

Now write $\varphi^{\prime \prime}+\left(d A^{\prime}-B^{\prime}\right) \varphi^{\prime}=\nu_{1} \varphi^{\prime 2}+\nu_{2} \varphi^{\prime} A^{\prime}$. This is consistent with the above if $\nu_{1}=\frac{1}{2}\left(a_{2}+a_{1}\right)$, and $\nu_{2}=(D-1)+\frac{1}{2}(D-2) a_{1} a_{2}$. We can choose $B$ at will by making coordinate transformations of $y$, so we may choose $B=\left(d-\nu_{2}\right) A=-1 / 2(D-1) a_{1} a_{2} A$. This then implies $\varphi^{\prime \prime}=\nu_{1} \varphi^{\prime 2}$. Thus the domain-wall solution is given by

$$
\begin{aligned}
e^{-\frac{1}{2}\left(a_{1}+a_{2}\right) \varphi} & =H \\
e^{\frac{1}{2}(D-2) a_{1} a_{2} A} & =e^{-B}=\tilde{b}_{1} H^{\frac{a_{2}}{a_{2}+a_{1}}}+\tilde{b}_{2} H^{\frac{a_{1}}{a_{2}+a_{1}}}
\end{aligned}
$$

where $H$ is the harmonic function $H=e^{-\frac{1}{2}\left(a_{1}+a_{2}\right) \varphi_{0}}+k|y|$, and $\tilde{b}_{i}=b_{i}\left(a_{1}+a_{2}\right) /(2 k)$. If $b_{1}$ and $b_{2}$ are of opposite signs, we can take a limit of $k \rightarrow 0$, in which the dilaton becomes a constant, given by

$$
e^{\left(a_{1}-a_{2}\right) \varphi}=e^{\left(a_{1}-a_{2}\right) \varphi_{\mathrm{AdS}}}=-\frac{a_{2}^{2} g_{2}^{2}\left[(D-2) a_{1}^{2}-2(D-1)\right]}{a_{1}^{2} g_{1}^{2}\left[(D-2) a_{2}^{2}-2(D-1)\right]} .
$$

The metric then becomes $\operatorname{AdS}_{D}$. This can be easily seen since in this case, we have $A \sim$ $\log |y|$ and $e^{2 B} \sim y^{-2}$. In all the examples in our paper, we have $a_{1}>a_{2}$. Different situations arise according to whether $\varphi_{0}$ is chosen to be less than, greater than, or equal to, $\varphi_{A d S}$. The solution is real provided that $b_{2}>b_{1}$. Except when $\varphi_{0}=\varphi_{A d S}$, there is a delta-function curvature singularity at $y=0$, since the harmonic function is taken to depend on $|y|$. One can then think of $y=0$ as defining a domain wall, separating the two regions of spacetime with $y>0$ and $y<0$. For $k>0$, the solution is real for all values of $y$, and the metric is singular at $|y|=\infty$, where its behaviour is dominated by the 
contribution of the $g_{2}$ cosmological term. By contrast, for $k<0$ the solution is real for

$|y|<y_{0} \equiv e^{-\frac{1}{2}\left(a_{1}+a_{2}\right) \varphi_{0}}$, and the metric is singular at $y_{0}$, where its behaviour is dominated by the $g_{1}$ cosmological term. In both cases the solution near the horizon at $y=0$ describes two reflection-symmetric portions of spacetime. If $\varphi_{0}=\varphi_{A d S}$, then the metric becomes the AdS metric at $y=0$. If $k>0$ and $\varphi_{0}>\varphi_{A d S}$, or $k<0$ and $\varphi_{0}<\varphi_{A d S}$, the metrics on the two sides of $y=0$ reach the AdS form for some value of $|y|$ that is greater than zero. On the other hand, if $k>0$ and $\varphi_{0}<\varphi_{A d S}$, or $k<0$ and $\varphi_{0}>\varphi_{A d S}$, the metrics on the two sides will not have reached the AdS form when they join at $y=0$.

\section{A general class of cosmological instantons}

We consider a general class of Lagrangian in $D$-dimensions of the form given in (B.1) and (B.2) with $\lambda=0$, i.e.

$$
e^{-1} \mathcal{L}=R-\frac{1}{2}(\partial \varphi)^{2}-V(\varphi)
$$

where

$$
V(\varphi)=\frac{1}{2} g_{1}^{2} e^{a_{1} \varphi}-\frac{1}{2} g_{2}^{2} e^{a_{2} \varphi}
$$

and dilaton coupling constants $a_{1}$ and $a_{2}$ satisfy

$$
a_{1} a_{2}=\frac{2(D-1)}{D-2} .
$$

Thus without loss of generality, we can assume that both $a_{1}$ and $a_{2}$ are positive. This scalar potential arises as breathing mode of spherically reduction of supergravities with admits AdS $\times$ Sphere solution. For example, M-theory reduction on $S^{7}, S^{4}$, Type IIB string on $S^{5}$ and self-dual three string on $S^{3}$. In particular, in Minkowskian signature these theories all have $a_{1}>a_{2}$. The potential has the following properties

$$
\begin{aligned}
V\left(\varphi_{0}\right) & =0, \quad \text { for } \quad e^{\left(a_{1}-a_{2}\right) \varphi_{0}}=\frac{g_{2}^{2}}{g_{1}^{2}} \\
V_{, \varphi_{0}} & =\frac{1}{2}\left(a_{1}-a_{2}\right) g_{1}^{-\frac{2 a_{2}}{a_{1}-a_{2}} g_{2}^{\frac{2 a_{1}}{a_{1}-a_{2}}}}, \\
V_{, \varphi}\left(\varphi_{\mathrm{AdS}}\right) & =0, \quad \text { for } \quad e^{\left(a_{1}-a_{2}\right) \varphi_{\mathrm{AdS}}}=\frac{a_{2} g_{2}^{2}}{a_{1} g_{2}^{2}}, \\
V_{\mathrm{AdS}}=V\left(\varphi_{\mathrm{AdS}}\right) & =\frac{1}{2}\left(a_{2}-a_{1}\right)\left(g_{2}^{2} / a_{1}\right)^{\frac{a_{1}}{a_{1}-a_{2}}}\left(g_{1}^{2} / a_{2}\right)^{-\frac{a_{2}}{a_{1}-a_{2}}} .
\end{aligned}
$$

Note that we always have $\varphi_{\text {AdS }}<\varphi_{0}$.

As in the case of $D=4$ discussed in section 4.1, the solution is better analysed in Euclidean-signatured space, and thus the metric ansatz we have for the instanton is of the 
form

$$
d s^{2}=d \sigma^{2}+b(\sigma)^{2} d \Omega^{2}
$$

The scalar $\varphi$ also depends only on $\sigma$. The equations of motion are then given by

$$
\varphi^{\prime \prime}+(D-1) \varphi^{\prime} \frac{b^{\prime}}{b}=V_{, \varphi}(\varphi), \quad b^{\prime \prime}=-\frac{1}{(D-2)(D-1)} b\left(\varphi^{\prime 2}+V(\varphi)\right)
$$

together with the first-order constraint

$$
\left(\frac{b^{\prime}}{b}\right)^{2}-\frac{1}{b^{2}}=\frac{1}{(D-2)(D-1)}\left(\frac{1}{2} \varphi^{\prime 2}-V(\varphi)\right) .
$$

Following [1], we assume at the initial time $\sigma=0$ that $\varphi(0)=\varphi_{0}$, and hence the potential $V(\varphi)$ vanishes, and also that $\varphi^{\prime}(0)=0$. Then at small values of $\sigma$, the space is purely Euclidean, with $b=v_{0} \sigma$, where $v_{0}$ is the initial velocity. The evolutionary structure depends on whether $a_{1}>a_{2}$, or $a_{1}<a_{2}$, corresponding to the two cases discussed in sections 4.1 and 4.3 for $D=4$.

$\underline{\text { Case (1): } a_{1}>a_{2}}$

In this case, from (C.4) we see that $V_{, \varphi}\left(\varphi_{0}\right)>0$, so the dilaton field will be driven towards positive infinity, and it will not stabilise at $\varphi=\varphi_{\text {AdS }}$, since $\varphi_{\text {AdS }}<\varphi_{0}$. The negative acceleration $b^{\prime \prime}$ will eventually reverse the sign of the velocity, and the universe will end up at a singularity at finite $\sigma_{f}$. At large value of $\varphi$, we have $V(\varphi) \sim \frac{1}{2} g_{1}^{2} e^{a_{1} \varphi}$, and if $a_{1}>a_{\text {crit }}$ the potential contribution is less divergent than the other terms in the equations of motion, and hence the solution becomes

$$
b \sim\left(\sigma_{f}-\sigma\right)^{\frac{1}{D-1}}, \quad \varphi^{\prime} \sim \frac{D-2}{\sqrt{D-1}}\left(\sigma_{f}-\sigma\right)^{-1} .
$$

Substituting this solution into the potential, it straightforward to show that

$$
a_{\text {crit }}^{2}=\frac{4(D-1)}{(D-2)^{2}}
$$

Thus for our set of dilaton coupling constants $a_{1}, a_{2}$, satisfying (C.3), we have $a_{1} a_{2}>a_{\text {crit }}^{2}$. (Note that in $D=4$, we have $a_{1} a_{2}=a_{\text {crit. }}$ ) Thus in all our cases, we have $a_{1}>a_{\text {crit. }}$. The late-time solution can then be obtained by making the following ansatz

$$
b \sim\left(\sigma_{f}-\sigma\right)^{b_{1}}, \quad e^{\frac{1}{2} a_{1} \varphi} \sim \frac{b_{2}}{\sigma_{f}-\sigma},
$$

where the constants $b_{1}$ and $b_{2}$ can be determined by substituting this ansatz into the equations of motion. 
$\underline{\text { Case }(2): a_{1}<a_{2}}$

In this case, we have $V_{, \varphi}\left(\varphi_{0}\right)<0$, so the dilaton will be driven towards left. It will either stabilise at $\varphi=\varphi_{\text {AdS }}$, or else $\varphi$ will head towards $-\infty$, depending on the initial conditions. Note that $V(\varphi)$ does not monotonically increase in the region; it has a maximum value $V_{\text {AdS }}$. Two possible scenarios emerge: if the potential is mild, then the dilaton will stabilise at $\varphi=\varphi_{\text {AdS }}$, and the solution will be described by an AdS Lagrangian $e^{-1} \mathcal{L}=R-V_{\text {AdS }}$, with solution given by

$$
b \sim \sin \left(\sqrt{\frac{V_{\mathrm{AdS}}}{(D-2)(D-1)}}\left(\sigma_{f}-\sigma\right)\right) .
$$

Note that in this case, the solution has no singularity, and the universe smoothly shrinks back to zero size, and the metric at $\sigma \rightarrow \sigma_{f}$ is again purely Euclidean. The universe afterwards become periodic. Of course, if the sloop of the potential is sharp, then the dilaton will be driven to infinity, and the universe will end up at a singularity, with the solution near $\sigma_{f}$ of the form (C.9).

\section{Curvatures for instanton and cosmological metrics}

We look for solutions in $D$ dimensions where the metric has Euclidean or Minkowskian signature, and takes the form

$$
\begin{aligned}
\text { Euclidean : } & d s^{2}=d \sigma^{2}+b(\sigma)^{2} d \Omega^{2}, \\
\text { Minkowskian : } & d s^{2}=-d \tau^{2}+b(\tau)^{2} d \Omega^{2},
\end{aligned}
$$

where $d \Omega^{2}$ is the metric on the unit $n$-sphere. In the obvious orthonormal basis $\hat{e}^{0}=d \sigma$ (or $\left.\hat{e}^{0}=d \tau\right), \hat{e}^{i}=b e^{i}$, we find that the vielbein components of the Riemann tensor are given by

$$
\begin{aligned}
\text { Euclidean : } \quad \hat{R}_{j k \ell}^{i} & =\frac{1}{b^{2}} R_{j k \ell}^{i}-\left(\frac{b^{\prime}}{b}\right)^{2}\left(\delta_{k}^{i} \delta_{j \ell}-\delta_{\ell}^{i} \delta_{j k}\right), \\
\hat{R}_{0 j 0}^{i} & =-\frac{b^{\prime \prime}}{b} \delta_{j}^{i} \\
\text { Minkowskian : } \quad \hat{R}_{j k \ell}^{i} & =\frac{1}{b^{2}} R_{j k \ell}^{i}+\left(\frac{\dot{b}}{b}\right)^{2}\left(\delta_{k}^{i} \delta_{j \ell}-\delta_{\ell}^{i} \delta_{j k}\right), \\
\hat{R}_{0 j 0}^{i} & =-\frac{\ddot{b}}{b} \delta_{j}^{i} .
\end{aligned}
$$

(This is in fact true for an arbitrary metric $d \Omega^{2}$; it need not yet be specified to be a unit sphere.) Thus we find that the vielbein components of the Ricci tensor are given by

$$
\text { Euclidean : } \quad \hat{R}_{i j}=\frac{1}{b^{2}}\left((n-1)\left(1-b^{2}\right)-b b^{\prime \prime}\right) \delta_{i j}
$$




$$
\hat{R}_{00}=-n \frac{b^{\prime \prime}}{b}
$$

$$
\begin{aligned}
\text { Minkowskian : } \quad \hat{R}_{i j} & =\frac{1}{b^{2}}\left((n-1)\left(1+\dot{b}^{2}\right)+b \ddot{b}\right) \delta_{i j}, \\
\hat{R}_{00} & =-n \frac{\ddot{b}}{b},
\end{aligned}
$$

where we have now substituted that the metric $d \Omega^{2}$ is for the unit $n$-sphere, whose its Ricci

tensor is $R_{i j}=(n-1) \delta_{i j}$. (In fact the Ricci tensor $\hat{R}_{a b}$ will take the same form for any Einstein metric $d \Omega^{2}$ normalised to have Ricci tensor $R_{i j}=(n-1) \delta_{i j}$.)

\section{E Effective cosmological constants in sphere reductions}

Although it is not directly relevant to our calculations in this paper, it is perhaps worthwhile to investigate the form of the effective cosmological constant that one obtains in a sphere reduction, if the breathing mode scalar is eliminated using its equation of motion. This result is, however, relevant in the model of Hawking and Turok [4], which is inspired by the 7-sphere reduction of $D=11$ supergravity. They consider a four-dimensional Lagrangian where the breathing mode does not appear, and has evidently been integrated out. (This can be seen from the fact that although there is an $F_{(4)}^{2}$ term in their Lagrangian, it is not multiplied by the exponential of the breathing-mode scalar, unlike the situation in (2.3).) The inflaton scalar in the Lagrangian in [ [] is presumably, therefore, to be thought of as one of the other scalars coming from the $S^{7}$ reduction of $D=11$ supergravity. One of the other features of the Lagrangian in 四 is that the sign of the kinetic term for $F_{(4)}^{2}$ is the opposite of the one that is directly inherited from $D=11$ under dimensional reduction. The explanation for this sign reversal is implicitly contained in [15], although they do not discuss the effective lower-dimensional Lagrangian. Using the results obtained in this paper, we can present a more explicit demonstration of this.

Consider the four-dimensional Minkowski-signature Lagrangian (2.3), which includes the breathing-mode scalar $\varphi$. As we have observed, there exist solutions where $\varphi=\varphi_{A d S}$ is a constant, which, in terms of the (constant) antisymmetric tensor $F_{(4)}$ is given by

$$
R_{7}=-\frac{7}{144} e^{-\frac{60}{7} \alpha \varphi_{A d S}} F_{(4)}^{2},
$$

as can be seen from (2.4b). Substituting this into the equation of motion (2.4a) for the metric, one finds

$$
R_{\mu \nu}=-\frac{1}{9} e^{-6 \alpha \varphi_{A d S}}\left(F_{(4) \mu \nu}^{2}-\frac{3}{8} F_{(4)}^{2} g_{\mu \nu}\right)
$$




$$
=-\frac{2}{7} e^{\frac{18}{7} \alpha \varphi_{A d S}} R_{7} g_{\mu \nu}
$$

Thus we see that indeed $R_{\mu \nu}$ is a negative multiple of $g_{\mu \nu}$, as one expects from the FreundRubin $\mathrm{AdS}_{4} \times S^{7}$ vacuum solution.

If one were to derive (E.2) from a Lagrangian involving only the metric and some 4-index antisymmetric tensor field strength $H_{(4)}$, then this Lagrangian would have to have the form

$$
e^{-1} \mathcal{L}=R+\frac{1}{48} H_{(4)}^{2}
$$

with a positive, rather than negative, sign for the kinetic term for $H_{(4)}$. Comparing the equations of motion from this Lagrangian with (E.2), we obtain agreement if we choose

$$
H_{(4)}=\frac{2}{\sqrt{3}} e^{-3 \alpha \varphi_{A d S}} F_{(4)},
$$

where, however, one still needs to take into account the relation (E.1).

Although in this sense one can say that the effect of the 7-sphere compactification of $D=11$ supergravity is to reverse the sign of the kinetic term for the 4 -form field strength, this viewpoint strikes us as somewhat artificial, since $H_{(4)}$ appearing in (E.3) is not the same as the 4-form field strength $F_{(4)}$ of $D=11$ supergravity. At the classical level the two are related by (E.4), but at the quantum level it might be more appropriate to retain both the original supergravity $F_{(4)}$ and the breathing mode $\varphi$ in deriving the dynamics of the inflaton field. If one wants to take seriously the proposition that the inflationary mechanism follows from $D=11$ supergravity, then the inflaton considered in [4] must be one of the scalar fields coming from the $S^{7}$ reduction. Even at the classical level, the interactions of the inflaton will involve the original supergravity 4 -form $F_{(4)}$, the breathing mode $\varphi$, and the scalar curvature $R_{7}$ of the 7 -sphere. It would therefore be misleading to think of the field strength $H_{(4)}$ in the effective Lagrangian (E.3) as being synonymous with the true supergravity field strength $F_{(4)}$. 


\section{References}

[1] S.W. Hawking and N. Turok, Open inflation without false vacua, hep-th/9802030.

[2] A. Linde, Quantum creation of an open inflationary universe, gr-qc/9802038.

[3] S.W. Hawking and N. Turok, Comment on 'Quantum creation of an open universe,' by Andrei Linde, gr-qc/9802062.

[4] S.W. Hawking and N. Turok, Open inflation, the four form and the cosmological constant, hep-th/99803156.

[5] W. Unruh, On the Hawking-Turok solution to the open universe wave function, grqc/9803050.

[6] R. Bousso and A. Linde, Quantum creation of a universe with $\Omega \neq 1$ : singular and non-singular instantons, gr-qc/9803068.

[7] J. Garriga, Open inflation and the singular boundary, hep-th/98030210.

[8] A. Vilenkin, Singular instantons and creation of open universes, hep-th/9803084.

[9] A. Vilenkin, The wave function discord, gr-qc/9804051.

[10] J. Garriga, Smooth "creation" of an open universe in five dimensions, hep-th/98040106.

[11] P.F. Gonzalez-Diaz, Non-singular instantons for the creation of open universes, hepth/9805012.

[12] P.M. Saffin, A. Mazumbdar and E.J. Copeland, Instantons from low energy string actions, hep-th/9805032.

[13] M.J. Duff and P. van Nieuwenhuizen, Quantum inequivalence of different field representations, Phys. Lett. B94 (1980) 179 .

[14] A. Aurelia, H. Nicolai and P.K. Townsend, Hidden constants: the theta parameter of $Q C D$ and the cosmological constant of $N=8$ supergravity, Nucl. Phys. B176 (1980) 509.

[15] P.G.O. Freund and M.A. Rubin, Dynamics of dimensional reduction, Phys. Lett. B97 (1980) 233. 
[16] S.W. Hawking, The cosmological constant is probably zero, Phys. Lett. B134, (1984) 403.

[17] J.D. Brown and C. Teitelboim, Neutralisation of the cosmological constant by membrane creation, Nucl. Phys. B297 (1988) 787.

[18] M.J. Duff, The cosmological constant is possibly zero, but the proof is probably wrong, Phys. Lett. B226 (1989) 36.

[19] M.J. Duncan and L.G. Larsen, Four forms and the vanishing of the cosmological constant, Nucl. Phys. B336 (1990) 100.

[20] M.J. Duff and C.N. Pope, Consistent truncations in Kaluza-Klein theories, Nucl. Phys. B255 (1985) 355.

[21] M.J. Duff, B.E.W. Nilsson and C.N. Pope, Kaluza-Klein supergravity, Phys. Rep. 130 (1986) 1.

[22] C.N. Pope and K.S. Stelle, Zilch currents, supersymmetry and Kaluza-Klein consistency, Phys. Lett. B198 (1987) 151.

[23] M.A. Awada, M.J. Duff and C.N. Pope, $N=8$ supergravity breaks down to $N=1$, Phys. Rev. Lett. 50 (1983) 294.

[24] D.N. Page, Classical stability of round and squashed seven-spheres in eleven-dimensional supergravity, Phys. Rev. D28 (1983) 2976.

[25] M.J. Duff, H. Lü and C.N. Pope, Supersymmetry without supersymmetry, Phys. Lett. B409 (1997) 136, hep-th/9704186.

[26] J.H. Schwarz, Covariant field equations of chiral $N=2 D=10$ supergravity, Nucl. Phys. B226 (1983) 269. See also J.H. Schwarz and P. West, Symmetries and transformations of chiral $N=2 D=10$ supergravity, Phys. Lett. B126 (1983) 301; P. Howe and P. West, The complete $N=2 D=10$ supergravity, Nucl. Phys. B238 (1984) 181.

[27] E. Cremmer, H. Lü, C.N. Pope and K.S. Stelle, Spectrum generating symmetries for BPS solitons, Nucl. Phys. B520 (1998) 132, hep-th/9707207.

[28] M. Cvetic and H.H. Soleng, Supergravity domain walls, Phys. Rep. 282 (1997) 159, hep-th/9604090. 
[29] E. Cremmer, B. Julia and J. Scherk, Supergravity theory in 11 dimensions, Phys. Lett. B76 (1978) 409.

[30] M.J. Duff and C.N. Pope, Kaluza-Klein supergravity and the seven sphere, in Supersymmetry and Supergravity 82 , proceedings of the Trieste conference.

[31] M.J. Duff, G.W. Gibbons and P.K. Townsend, Macroscopic superstrings as interpolating solitons, Phys. Lett. B332 (1994) 321, hep-th/9405124.

[32] M.J. Duff and K.S. Stelle, Multimembrane solutions of $D=11$ supergravity, Phys. Lett. B253 (1991) 113.

[33] E. Bergshoeff, M. de Roo, M.B. Green, G. Papadopoulos and P.K. Townsend, Duality of type II 7-branes and 8-branes, Nucl. Phys. B470 (1996) 113, hep-th/9601150;

P.M. Cowdall, H. Lü, C.N. Pope, K.S. Stelle and P.K. Townsend, Domain walls in massive supergravities, Nucl. Phys. B486 (1997) 49, hep-th/9608173.

[34] H. Lü and C.N. Pope, Domain walls from M-branes, Mod. Phys. Lett. A12 (1997) 1087, hep-th/9611079.

[35] M.J. Duff and C.J. Isham, Self-duality, helicity and supersymmetry: the scattering of light by light, Phys. Lett. B86 (1979) 157 .

[36] M.J. Duff and C.J. Isham, Self-duality, helicity and coherent states in non-abelian gauge theories, Nucl. Phys. B162 (1980) 271.

[37] S.W. Hawking and S.F. Ross, Duality between electric and magnetic black holes, Phys. Rev. D52 (1995) 5865, hep-th/9504019.

[38] M.J. Duff and J. Madore, Einstein-Yang-Mills pseudoparticles and electric charge quantization, Phys. Rev. D18 (1978) 2788 .

[39] A.S. Belavin, A.M. Polyakov, A.S. Schwartz and Y.S. Tyupkin, Pseudoparticle solutions of the Yang-Mills equations, Phys. Lett. B59 (1975) 85.

[40] G. 't Hooft, Symmetry breaking through Bell-Jackiw anomalies, Phys. Rev. Lett. 37 (1976) 8 .

[41] R. Bousso and A. Chamblin, Open inflation from non-singular instantons: wrapping the universe with a membrane, hep-th/9805167. 
[42] E. Bergshoeff, E. Sezgin and P.K. Townsend, Supermembranes and eleven-dimensional supergravity, Phys. Lett. B189 (1987) 75.

[43] M.J. Duff, J.T. Liu and R. Minasian, Eleven-dimensional origin of string/string duality: a one-loop test, Nucl. Phys. B452 (1995) 261, hep-th/9506126.

[44] J.H. Schwarz, The power of M-theory, Phys. Lett. B367 (1996) 97, hep-th/9510086.

[45] S.P. de Alwis, A note on brane tension and M theory, Phys. Lett. B388 (1996) 291, hep-th/9607011.

[46] M.S. Bremer, H. Lü, C.N. Pope and K.S. Stelle, Dirac quantisation conditions and Kaluza-Klein reduction, hep-th/9710244, to appear in Nucl. Phys. B.

[47] G. Neugebaur and D. Kramer, Ann. der Physik (Leipzig) 24 (1969) 62;

P. Breitenlohner, D. Maison and G.W. Gibbons, Four-dimensional black holes from Kaluza-Klein theories, Comm. Math. Phys. 120 (1988) 253;

G. Clement and D.V. Gal'tsov, Stationary BPS solutions to dilaton-axion gravity, Phys. Rev. D54 (1996) 6136, hep-th/9607043;

D.V. Gal'tsov and O.A. Rytchkov, Generating branes via sigma-models, hep-th/9801160;

K.S. Stelle, BPS branes in supergravity, hep-th/9803116;

C.M. Hull and B. Julia, Duality and moduli spaces for time-like reductions, hepth/9803239.

[48] E. Cremmer, I.V. Lavrinenko, H. Lü, C.N. Pope, K.S. Stelle and T.A. Tran, Euclideansignature supergravities, dualities and instantons, hep-th/9803259, to appear in Nucl. Phys. B.

[49] H. Lü, C.N. Pope, E. Sezgin and K.S. Stelle, Dilatonic p-brane solitons, Phys. Lett. B371 (1996) 46, hep-th/9511203.

[50] P.K. Townsend and P. van Nieuwenhuizen, Gauged seven-dimensional supergravity, Phys. Lett. B125 (1983) 41.

[51] L.J. Romans, The $F(4)$ gauged supergravity in six dimensions, Nucl. Phys. B269 (1986) 691.

[52] S.W. Hawking and H.S. Reall, Inflation, Singular Instantons and Eleven Dimensional Cosmology, hep-th/9807100. 